\title{
Dry patches in a flowing film: Predicting rewetting, and the effects of inertia.
}

\author{
J. Sebilleau, ${ }^{1,2}$ L. Lebon, ${ }^{1}$ and L. Limat ${ }^{1}$ \\ ${ }^{1}$ Matières et Systèmes Complexes (MSC), Université Paris Diderot, CNRS - UMR 705\%, \\ Bâtiment Condorcet, 10 rue Alice Domon et Léonie Duquet, 75013 Paris, France \\ ${ }^{2}$ INPT, UPS, IMFT (Institut de Mécanique des Fluides de \\ Toulouse), Université de Toulouse, Allée Camille Soula, F-31400 \\ Toulouse, France and CNRS, IMFT, F-31400 Toulouse, France
}

\begin{abstract}
We study the effects of inertia on the shape and stability of dry patches using liquids of decreasing viscosities. These dry patches are formed when a liquid film flows down along a substrate under partial wetting conditions. They become stationary and exhibit an "arch" shape well described by a simple viscous model developed long ago by Podgorski. Surprisingly, this "arch" shape appears to be robust when one decreases the fluid viscosity which increases inertial effects, but the evolution of the apex curvature upon flow rate is strongly affected. We here proposed an improved description of the dry patch evolution taking into account several physical effect as the hydrostatic pressure in the liquid film, the curvature of the contact line, and these inertial effects. These ones affect both the mechanical equilibrium of the rim surrounding the dry patch and the flow inside the rim. This model allows us to show that the dry patch shape remains extremely close to the viscous -Podgorski- prediction but with a rescaling of the apex curvature. It also allows us to get a better prediction of the apex curvature dependance upon flow rate and a prediction of the rewetting threshold above which dry patches are swept away by the film flow. This critical flow rate $\Gamma_{c}$ is in good agreement with our experiments performed in the range of of viscosity from 5 to $20 \mathrm{cP}$ with silicon oils.

PACS numbers:
\end{abstract}




\section{INTRODUCTION}

\section{A. History of the problem}

Wetting and dewetting phenomena have been intensively studied these least decades [16] but the complete description of these processes is still a challenge because of the subtle hydrodynamics in the vicinity of the contact line [7]. One challenging problem in this field is the stability of dry patches that can open spontaneously when a liquid film flows down an incline under partial wetting conditions. Such dry patches appear in numerous industrial situations where a liquid film flows on a poorly wettable surface ( coating, spreading, heat exchangers, desalination devices... ...), and have thus motivated several studies $[12-15,17-$ 22]. By analogy with the stability of a single hole in a static horizontal liquid film $[4,8-11]$ that closes when a critical thickness is exceeded, one could expect that the flow rate per unit length $\Gamma$ controls the stability of the dry zone and that above a critical flow rate $\Gamma_{c}$ the film will cover all the incline. As the dry patch is surrounded by a rim whose thickness must be of order of the capillary length $l_{c}=(\gamma / \rho g)^{1 / 2}$, where $\gamma$ is the surface tension, $\rho$ the density and $g$ the gravitational constant, and as the typical velocity of the advancing contact line must be of order the capillary velocity $U_{c}=\gamma / \eta$, where $\eta$ is the dynamic viscosity, one can expect this critical flow rate to be of order $\Gamma_{c} \sim U_{c} l_{c}$. Nevertheless this simple approach was not used in the first studies on the subject $[12,20]$.

Initially, Hartley et al. [12] proposed a prediction for the critical flow rate based on a balance between capillary forces and the downward momentum at the dry patch apex which yields:

$$
\Gamma_{c}=\frac{1}{3}\left(\frac{15}{2}\right)^{\frac{3}{5}}\left[\frac{\gamma(1-\cos \theta)}{\rho}\right]^{\frac{3}{5}}\left[\frac{\eta}{\rho g \sin \alpha}\right]^{\frac{1}{5}}
$$

in which we have corrected a $1 / 2$ erroneous factor and included the dependance upon $\alpha$ that was missing in [12]. In this model, the authors did not realize the existence of a rim surrounding the dry patch in which the liquid is collected. Their prediction is at least one order of magnitude higher than the first reproducible experiments performed only recently [13]. Later, S.D.R Wilson [20] investigated theoretically the flow near the apex by considering

the balance (normally to the contact line) between capillary forces, downward momentum carried by the film, weight of the rim and contact line curvature effect on the capillary forces. Assuming a parabolic shape of the dry patch near apex and estimating the fluid velocity in the rim, this author has calculated numerically the apex curvature evolution and the 
critical film thickness above which a dry patch becomes unstable. This pioneering approach, unfortunately predicts a radius of curvature at the apex that increases with the flow rate whereas, experimentally, this radius decreases [13-15]. As we shall see here, this unphysical behavior might be linked to the erroneous choice of an unstable branch of the curvature apex evolution.

More recently Podgorski et al. [13-15] performed the first reproducible experiments. They also developed models for the dry patch shape and the critical flow rate $\Gamma_{c}$. First, they investigated the dry patch shape and proposed a simple description in good agreement with their experiments [13]. This first model is based on a balance between capillary effects and the weight of the rim. They assumed that the cross section of the rim was an arc of circle and that the contact angle was uniform all along the contact line and equal to the static advancing contact angle. These assumption have been confirmed by optical measurements $[17,18]$ on dry patches studied for successive quasi static increases of the flow rate. A different approach has been developed by Wilson et al. [21] who studied similarity solutions of a slender dry patch in both weak and strong surface tension regimes. Their model, based on the lubrication approximation, allowed them to recover analytically the existence of the rim (in the strong surface tension case) but predicts a solution with an infinite slope at the contact line which is not relevant as lubrication approximation is used. In addition, the approximation $\psi<<1$ (see fig. 1) is not valid near the apex which makes questionable any prediction on the evolution of the radius of curvature at the apex. More recently Yatim et al. [22] have extended this approach to the case of steadily translating dry patches with negligible surface tension effects. Their similarity solutions present several physical features in common with dry patches studied experimentally as for example the existence of several possible dry patch width for a given imposed parameter here the speed of the dry patch. Nevertheless a precise comparison with experiments appears to be difficult as surface tension is neglected in this approach. Coming back to Podgorski description, assuming a Stokes flow in the rim and combining this flow with the mass conservation and the balance of forces normally to the contact line leads to a very simple prediction of the shape:

$$
\begin{aligned}
& x=R \frac{\cos \psi}{\sin ^{2} \psi} \\
& y=\frac{R}{3}\left(\frac{1-3 \cos ^{2} \psi}{\sin ^{3} \psi}-1\right)
\end{aligned}
$$


where $\psi$ is the angle between the tangent to the contact line and the direction of highest slope (see notations on fig. 1 ), and $R$ is the radius of curvature at the apex. The evolution

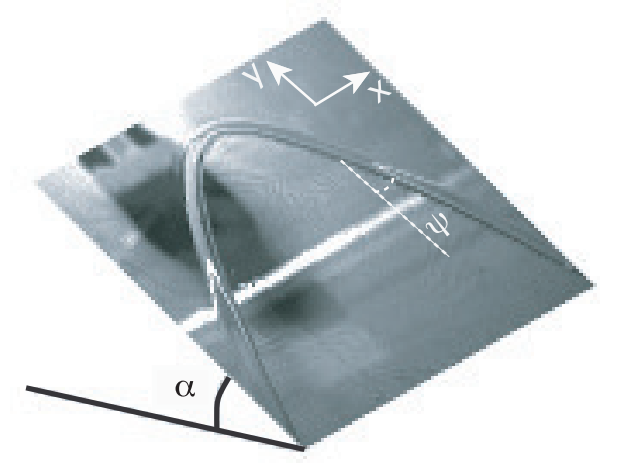

FIG. 1: Photo of a dry "arch" where some notations has been added.

of $R$ with the flow rate $\Gamma$ is given by:

$$
\begin{aligned}
R & =m f_{2}(\theta) \frac{l_{c}^{2}}{\sin \alpha} \frac{U_{c}}{\Gamma} \\
f_{2}(\theta) & =\frac{(1-\cos \theta)^{4}}{(\theta-\sin \theta \cos \theta)}
\end{aligned}
$$

where $m$ is a numerical factor taking into account the detail of the flow inside the rim. This parameter $m$ is not known exactly but a reasonable estimate $(m=0.23)$ can be obtained by identifying the flow inside the rim to something similar to the one inside a rivulet flowing along an incline in the lubrication approximation. This shape prediction is in very good agreement with experiments [13] but this model fails to reproduce all observed physical behaviors [15]. First, it predicts a stable solution for the radius of curvature $R$ at the apex, whatever could be the flow rate value, and thus completely misses the existence of a critical flow rate above which dry patches are not stable anymore. Also, the dependance of $R$ versus plate slope presents increasing discrepancies to the expected scaling $R \sim(\Gamma \sin \alpha)^{-1}$, when one looks to moderately inclined plates. To correct these issues, Podgorski et al. [15] proposed an improved model taking into account capillary effects linked to the contact line curvature that were missing in the balance of forces acting on the rim. This new model better predicts the dependance of $R$ with the plate slope and introduces a critical flow rate $\Gamma_{c}$ given by:

$$
\Gamma_{c}=\frac{6 \sqrt{3}}{25 \sqrt{5}} m H(\theta) \frac{l_{c} U_{c}}{\sqrt{\sin \alpha}}
$$


where $H(\theta)$ is a function of the contact angle given by:

$$
H(\theta)=\frac{(1-\cos \theta)^{9 / 2}}{(\theta-\sin \theta)(\theta-\sin \theta \cos \theta)^{1 / 2}}
$$

This prediction had the right order of magnitude but was not perfect: the predicted critical flow rate was two time larger than the experimental one in the case of a vertical wall and presented an unphysical divergence in the small slope limit.

\section{B. Outline of the paper}

In this paper, we present experiments performed with silicon oils of decreasing viscosities $\left(\eta=210^{-2}\right.$ Pa.s, $\eta=10^{-2}$ Pa.s and $\eta=510^{-3}$ Pa.s). These experiments are compared with a more complete model addressing both the shape and the stability of a dry patch. In this model, we first improve the balance of forces acting on the rim by taking into account capillary effects linked to the contact line curvature, hydrostatic pressure in the film, and inertial effects linked to momentum exchange between the film and the rim. This new balance of forces generalizes previous attempts [15], in which both the momentum and hydrostatic terms were missing. It leads to a prediction of the critical flow rate $\Gamma_{c}$ in good agreement with experiments [19] for sufficiently high viscosity (typically $\eta \sim 2.10^{-2} \mathrm{~Pa} . \mathrm{s}$ ). In a second step, as the agreement becomes not so good for less viscous oil, we consider also inertial effects in the flow inside the rim. Combining this improved estimation of the velocity in the rim with the new balance of force leads to an more complete description that is in good agreement with our experiments. This new model provides, for the first time to our knowledge, a reasonable prediction of the critical flow rate at moderate contact angle for any value of viscosity and surface tension.

We will first describe, in section 2, the experimental apparatus and report general observations. In section 3, we will present the first step of our modeling which consists in improving the balance of force acting on the rim. This leads to a theoretical prediction of the apex curvature evolution and of the critical flow rate that is compared to our experiments. In section 4, we improve the modeling of the flow inside the rim by considering inertial effects. Combining this improved model of the flow inside the rim with the more complete balance of force acting on the rim provides a more complete prediction that is compared to our experimental results. Finally, in section 5, we will give our conclusions and perspectives. 

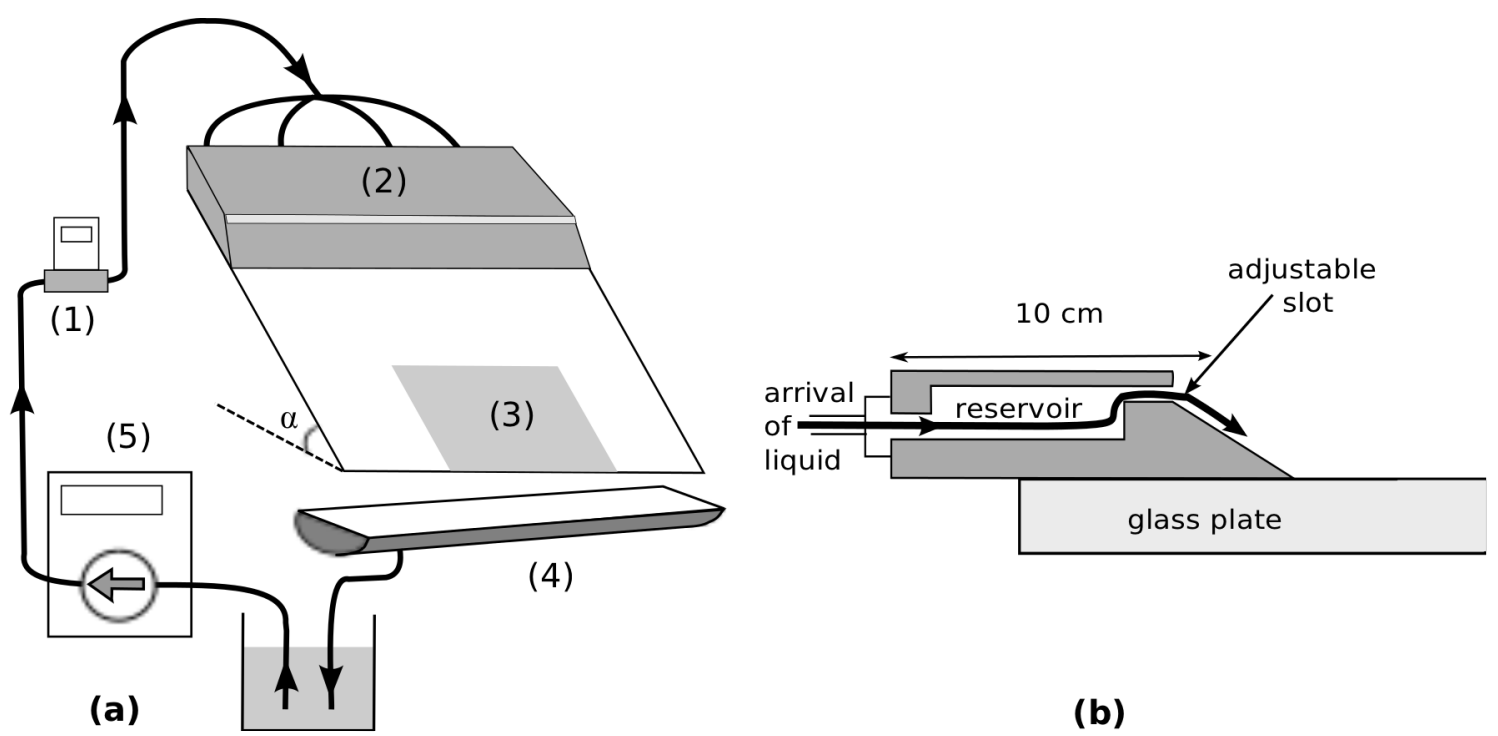

FIG. 2: (a): sketch of the experimental setup: the flow rate is measured by a flow meter (1) before the liquid reaches the slot injector (2). Then an homogenous liquid film flows down the inclined substrate which has been coated on its lower part (3). The liquid is then collected (4) and pumped again (5). (b): detailed sketch of the injector: this injector has been carefully design to ensure an homogenous liquid film flow on the incline.

\section{EXPERIMENTS AND GENERAL OBSERVATIONS}

\section{A. Experimental apparatus}

The experimental setup is described in fig. 2 (a). A uniform film flow of silicon oil is imposed on a glass plate (of size $40 \mathrm{~cm}$ x $40 \mathrm{~cm}$ ), which lower part exhibits partial wetting conditions (the details of the coating procedure are given below). The film is supplied by an injector, suggested on fig. 2 (b), across a thin slot of $40 \mathrm{~cm}$ length and of adjustable gap (gap height about $\simeq 0.5 \mathrm{~mm}$ ). This injector was carefully designed to ensure a uniform flow all along the slot. The flow uniformity has been checked by measuring the liquid film thickness with a needle first put in contact with the substrate and then with the free surface, the observed discrepancies were smaller than $2 \%$. The oil flows from the injector down the glass plate (and so flows over the partial wetting area) to a collector where it is pumped by a gear pump (Ismatec MC-Z). The flow rate is measured by a digital flow meter (Mc Millan 111 Flo-Meter). Three silicon oils (poly-dimethyl-siloxane PDMS) of different 


\begin{tabular}{|l|c|c|c|}
\hline & Rhodorsil 47V20 & HUBER M40.165.10 & Rhodorsil 47V5 \\
\hline kinematic viscosity $\nu\left(\mathrm{mm}^{2} / \mathrm{s}\right)$ & 18.5 & 11.33 & 5.19 \\
\hline specific mass $\rho\left(\mathrm{g} / \mathrm{cm}^{3}\right)$ & 0.95 & 1.05 & 0.922 \\
\hline surface tension $\gamma(\mathrm{mN} / \mathrm{m})$ & 20.6 & 20.5 & 20.5 \\
\hline dynamic viscosity $\eta=\rho \nu($ Pa.s $)$ & $1.7610^{-2}$ & $1.15510^{-2}$ & $4.610^{-2}$ \\
\hline
\end{tabular}

TABLE I: Physical properties of the silicon oils used in our experiments

dynamic viscosities have been used: Rhodorsil 47V20 of viscosity close to $20 \mathrm{cP}$, HUBER M40.165.10 of viscosity close to $10 \mathrm{cP}$ and Rhordorsil 47V25 of viscosity close to $5 \mathrm{cP}$. Their physical properties are given in Table I. In these conditions the total flow rate that can be supplied to the plate ranges between 0 and $100 \mathrm{~cm}^{3} \cdot \mathrm{s}^{-1}$, which implies a typical flow rate per unit length $\Gamma$ ranging between 0 and $2.5 \mathrm{~cm}^{2} \cdot \mathrm{s}^{-1}$. To obtain reproducible wetting conditions, the plate is first cleaned with sulfochromic acid and acetone. The upper part is left untreated to keep total wettability for silicon oils and a rectangular zone (about 20 $\mathrm{cm} \times 20 \mathrm{~cm}$ ) in the lower part is coated with a fluoropolymer (3M Fluorad FC725). This polymer ensures a partial wettability for silicon oils [16]. This treatment is different than the one (Steadlter lumocolor ink) used in [13]. It provides a higher contact angle and a smaller wetting hysteresis. A lot of care has to be taken during the coating to avoid inhomogeneities. Though rudimentary, our method gives satisfactory results even if this coating is fragile and has to be regularly renewed. The advancing and receding static contact angles are measured from drops deposited on the partial wetting area, the plate being kept horizontal. The volume of the drop is increased until the contact line begins to move, which gives us an estimate of the advancing contact angle. We found $\theta_{a} \simeq 52^{\circ}$ for Rhodorsil 47V20, HUBER and Rhodorsil 47V5 oils. A similar method, reducing now the volume, gives also the receding contact angle: $\theta_{r} \simeq 45^{\circ}$ for HUBER oil and $\theta_{r} \simeq 40^{\circ}$ for both Rhodorsil 47V20 and 47V5 oils.

\section{B. Qualitative observation of dry patches}

When the flow starts over the glass plate, the classical fingering instability occurs [23-25], and can lead to complex invasion scenarios of the partial wetting area. To avoid this 


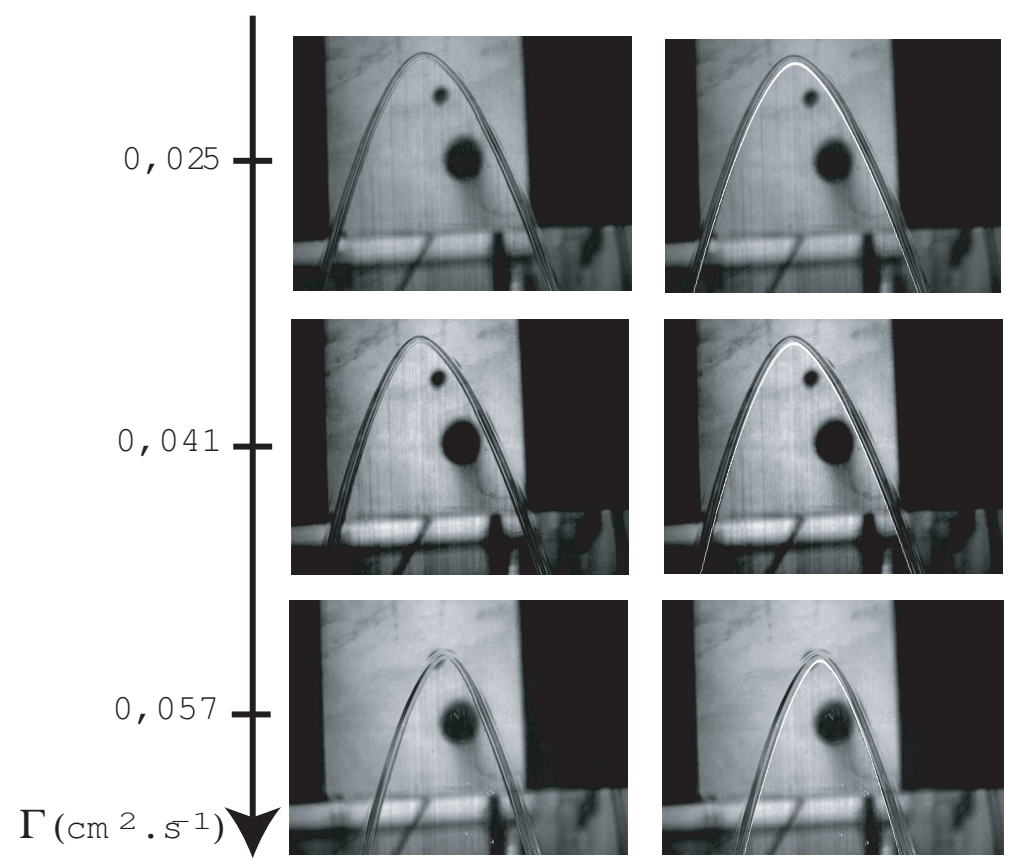

FIG. 3: Evolution of a "dry arch" observed for increasing values of the flow rate per unit length (oil HUBER M40.165.10), $\alpha=85^{\circ}$ ). On the right part of the figure, the shape is fitted by eq.(2)

phenomenon, a sufficiently large flow rate is first applied to cover the full plate with liquid. Then, the flow rate is decreased and one obtains a metastable film on the partial wetting area. Finally, a dry zone (see fig. 3) is initiated in the center of the coated area by a brief and localized air blow. When the final shape of "arch" is established, its evolution upon flow rate is studied as follows: the flow rate is slowly stepwise increased and the shape is recorded at each step. A typical evolution of the dry patch shape with the flow rate is reproduced on fig. 3. This isolated patch has the form of an arch and is edged by a rim which collects the liquid that would otherwise cover the dry surface. Therefore, the liquid film can be separated in two distinct regions: an upstream film of uniform thickness and uniform mean velocity, and a rim in which the flow is parallel to the contact line on the scale of the capillary length. We have tried to fit the contact line shape with Podgorski et al. model (eq. (2)) and found in all the case a nearly perfect fit. We have then used this fit yo extract the radius of curvature at the dry patch apex $R$. One can clearly see in both fig. 3 and 4 the dry zone tends to close when the flow rate is increased, and so the radius of curvature at the apex $R$ decreases with flow rate. This result is in contradiction with 


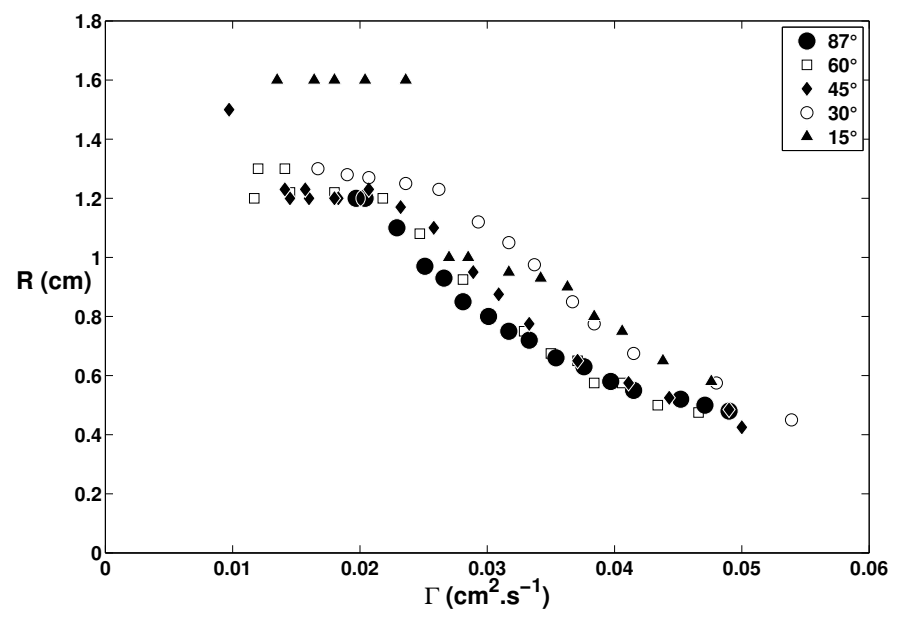

FIG. 4: Typical evolution of the radius of curvature at the apex versus the flow rate for the 47V20 Rhodorsil 47V20 oil. The radius of curvature at the apex $R$ has been measured by fitting the contact line with eq. (2).

S.D.R Wilson [20] modeling which predicts an increase of this radius of curvature when the flow rate increases. As said above, this unphysical behavior might be linked to an erroneous choice of an unstable branch of the radius of curvature at the apex in S.D.R Wilson modeling. The typical evolution of $R$ upon flow rate per unit length $\Gamma$ is reproduced on fig. 4 for several plate slopes $\alpha$. One can distinguish two successive stages in these curves. First, the radius $R$ remains constant, and then it decreases. As in previous papers on the subject, we attribute the first of these two regimes to the wetting hysteresis of the substrate. In the first stage, the contact angle evolves between $\theta_{r}$ and $\theta_{a}$ while the contact line remains pinned. In the second stage, the contact line evolves while keeping the contact angle uniform and nearly equal to $\theta_{a}$. This interpretation has been explicitly validated by Rio et al. $[17,18]$ with accurate optical contact angle measurements. The experiment stops when the critical flow rate is reached: when one reaches this upper limit, the contact line begins to slide down to produce a fully wet glass plate. Although this method allows to follow the evolution of the shape with the flow rate, it only gives an estimate of the critical flow rate $\Gamma_{c}$ because of eventual pinning of the contact line on defects of the partial wetting coated area. To obtain a more precise measurement of the critical flow rate, we try to open dry patches of various sizes and in different places at flow rate close to this upper limit. We record 
as the critical flow rate, the flow rate where all formed dry patches are unstable. These measurements have been performed for each oil and for different plate slopes $\alpha$ and will be discussed in the next sections (fig. 6,15 and 23) with the modeling of the critical flow rate.

\section{Comparison with previous models}

As told above, equations (2) found by Podgorski et al. [13] always provide an excellent fit of the observed patch shape. This is a strong result that suggests that this modeling is in some sense very "robust" and has captured essential features of the solution, but as we mentioned too, the question of the curvature radius selection at the apex is more puzzling. As we shall see, Podgorski modeling does not always give the right answer, which will impose to consider models of increasing complexity when both the plate inclination or the fluid viscosity are progressively decreased. The dependance of the radius of curvature at the apex $\mathrm{R}$ upon $1 /(\Gamma \sin \alpha)$ observed in our experiments is given on fig 5 for the three used silicon oils, and compared to the prediction (3), that appears as a bold line on these figures (a linear law is expected with this set of variables). As one can see, the agreement is very good (with no adjustable parameter, since $m$ is fixed at the value deduced from lubrication model) for both Rhodorsil 47V20 and Huber oil in the limit of a vertical plate, and for the points obtained in the limit of a contact angle saturating to the static advancing angle (as explained previously, the contact angle varies for the other points, on the "plateau" observed on the right). When the plate inclination is progressively reduced, the agreement still remains good but the expected straight line is slightly shift of a constant value that depends on the plate inclination. This effect has been explained and calculated in the second paper from Podgorski et al. [15], in which curvature effects of the contact line were added to the initial modeling. When one further reduces the plate inclination, even the slope of the expected line changes, and the agreement with both models from Podgorski become thus very poor. In the case of an even smaller viscosity (Rhodorsil 47V5), the agreement is very bad even for a vertical plate. Clearly, if one considers the three used oils, a decrease of the liquid viscosity introduces more and more discrepancies between experiment and Podgorski modeling.

Another quantity to consider is now the critical flow rate above which any dry patch becomes 

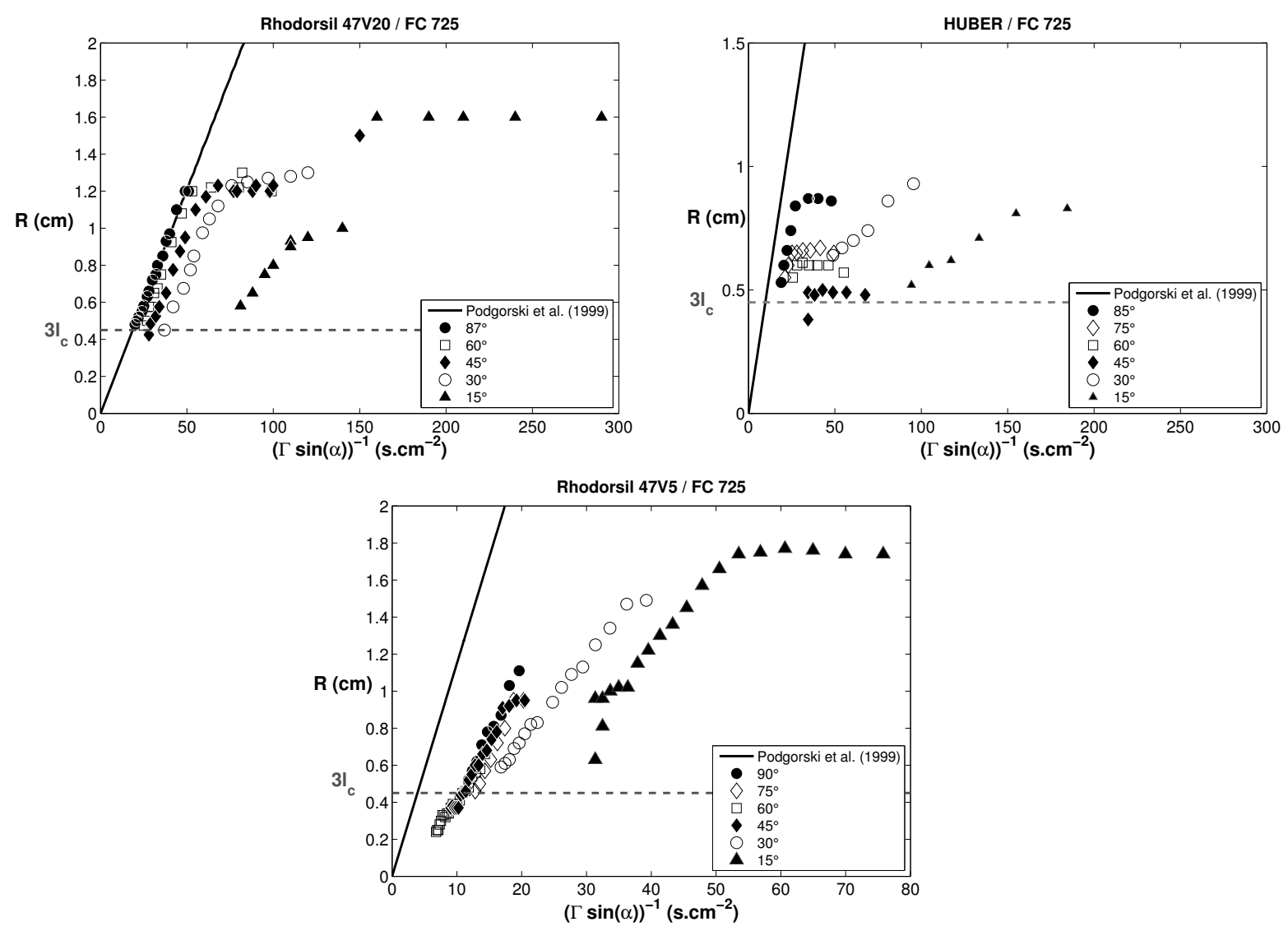

FIG. 5: Evolution of the radius of curvature at the apex with the flow rate. The dark line is the prediction (3) where we have taken $m=0.23$ and $\theta=\theta_{a}=52^{\circ}$.

unstable. Qualitatively, we observed experimentally that this critical flow rate was approximately reached when the curvature at the apex was of order of three capillary lengths, i.e $R \simeq 3 l_{c}$. This is clearly visible on fig. 5 , where all the curves are interrupted before to reach zero for a value of $\mathrm{R}$ of this order of magnitude. This finding is consistent with a qualitative idea proposed in the first paper from Podgorski [13], that this critical flow rate was reached when the contact line curvature was of order of the rim width (that is indeed of order of the capillary length). This means that curvature effects of the contact lines are essential ingredients that one has to take into account for calculating the critical flow rate, as attempted in the paper from S.D.R. Wilson [20] and in the second paper from Podgorski [15]. For a more quantitative comparison, and considering the fact that the model from S.D.R. Wilson fails to predict the evolution of the radius of curvature at the apex with the flow rate, we have compared on fig. 6 only the predictions of the second model from Podgorski, and also 

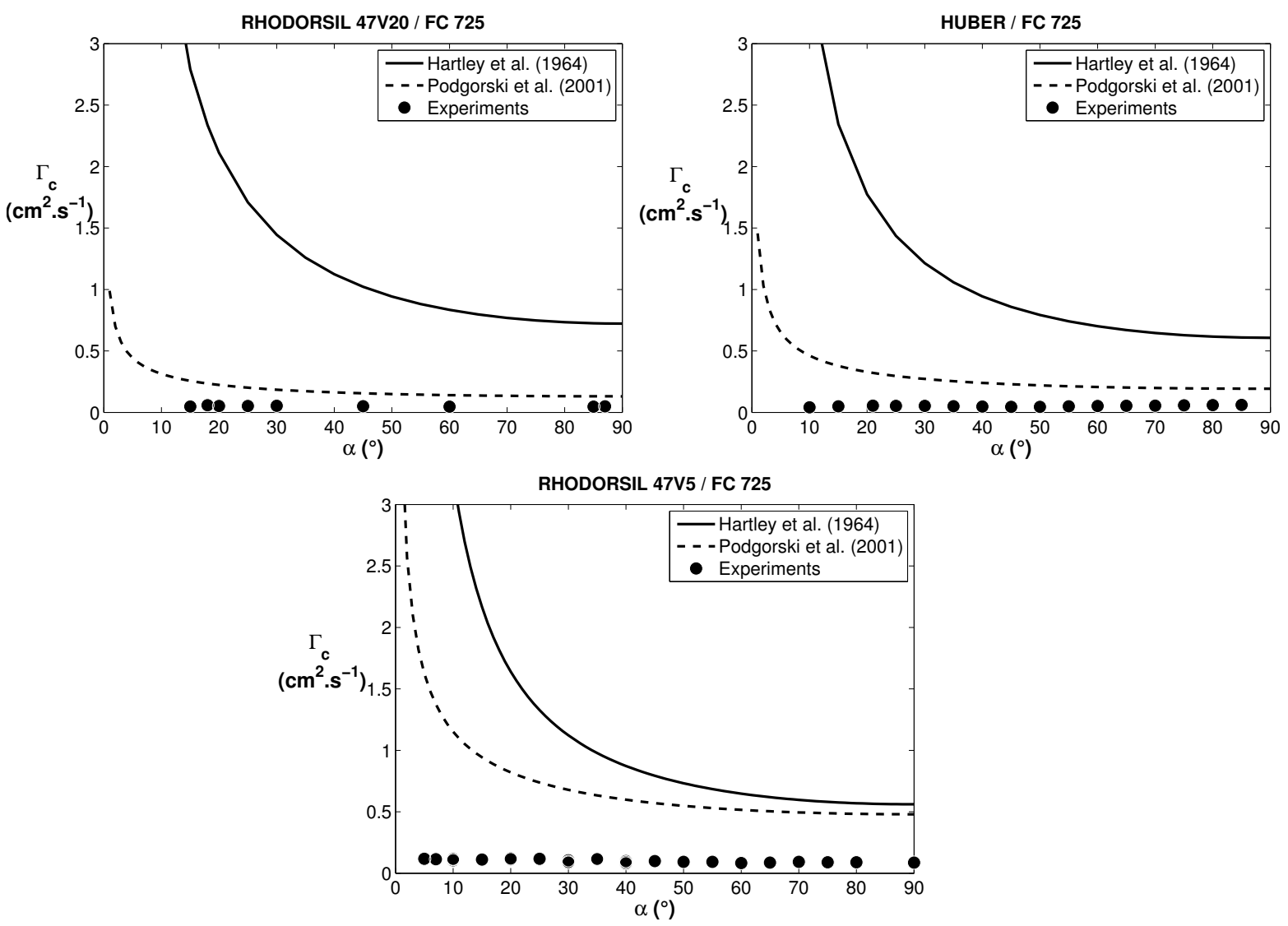

FIG. 6: Comparison of the measured critical flow rate with the theoretical predictions (1) and (4)

the initial guess from Hartley and Murgatroyd [12]. This one only considers a balance of inertia and capillarity, but has been a reference for many authors during a long time. As one can see on fig. 6, both curves fail to describe our data, with even a unphysical divergence of Podgorski modeling at low plate inclination. In addition Hartley and Murgatroyd gives a prediction that is more than ten times too large, while that of the second model of Podgorski has the right order of magnitude, but remains two or three times too large. Considering this preliminary comparison, it appears that the question of the shape and stability of dry patches is still an opened issue. Strictly speaking, the shape itself is well captured but the first model from Podgorski [13], but the length that scales this shape is not so well described. Only the case of a quasi-vertical plate, with a rather high fluid viscosity is well recovered by this simple modeling. The second modeling [15] is indeed a good improvement, as it allows one to understand the shift of the law governing the apex curvature with flow rate and the appearance of a critical flow rate, but the precise value of this critical flow rate, as 
well as the curvature behavior observed for weak plate inclination or low viscosities remain unsolved. These are the issues that we try to solve in the next sections by considering a more and more complex modeling.

\section{FIRST STEP : IMPROVING THE MECHANICAL EQUILIBRIUM OF THE RIM (MODEL 1)}

As said above, previous theories $[15,20]$ have shown qualitatively that the effect of the contact line curvature on the balance of forces acting on the rim is a necessary ingredient to predict the critical flow rate. As they however fail to predict quantitatively the apex curvature and the critical flow rate, we propose an improved balance of forces taking into account several effects neglected in these approaches: hydrostatic pressure in the liquid film and momentum exchange between the film and the rim. As we shall see, the hydrostatic pressure term will allow us at least to suppress the unphysical divergence when the plate slope become small.

\section{A. Order of magnitude}

As mentioned above, we distinguish two parts in the flow : the uniform film and the rim surrounding the dry area. The film flow is supposed to be homogenous and the liquid within the rim is assumed to flow in a direction parallel to the contact line. The cross section of rim free surface is supposed to be an arc of circle of radius $r$ enclosing an area $S$ (see fig. 7). Typical orders of magnitude for the flow rate per unit length $\Gamma$, thickness of the film $h$ and height of the rim $H$ have been measured on the experiment. From the value of $\Gamma$ and of $h$, one can easily derive the order of magnitude of the mean velocity of the fluid in the film $V=\Gamma / h$. The typical values of these quantities are given in table II. With these quantities, one can estimate typical dimensionless numbers for this problem, given in table III . These dimensionless numbers show that the flow in the film reduces to a Stokes flow driven by gravity and that the normal equilibrium of the contact line is mainly a balance between surface tension and the weight of the rim to which the others effects will be added as perturbations. 


\begin{tabular}{|c|c|}
\hline Flow property & typical value \\
\hline flow rate & $\Gamma \sim 0-0.05\left(\mathrm{~cm}^{2} . \mathrm{s}^{-1}\right)$ \\
\hline film thickness & $h \sim 0.2(\mathrm{~mm})$ \\
\hline fluid velocity in the film & $V \sim 0-2\left(\mathrm{~cm}^{-} \mathrm{s}^{-1}\right)$ \\
\hline rim height & $H \sim 1(\mathrm{~mm})$ \\
\hline
\end{tabular}

TABLE II: Typical scales of the flow in our experiments

\begin{tabular}{|c|c|}
\hline Dimensionless number & typical value \\
\hline Reynolds number $R e=\Gamma / \nu$ & $R e \sim 0-0.5$ \\
\hline Capillary number $C a=\eta V / \gamma$ & $C a \sim 10^{-3}$ \\
\hline Bond number $B o=\rho g H^{2} / \gamma$ & $B o \sim 0.5$ \\
\hline Weber number $W e=\rho V^{2} h / \gamma$ & $W e<0.06$ \\
\hline Froude number $F r=V^{2} h / g H^{2}$ & $F r<0.06$ \\
\hline Morton number $M=\rho \gamma^{3} / \eta^{4} g$ & $10^{4}$ \\
\hline
\end{tabular}

TABLE III: Typical dimensionless numbers

\section{B. Mass conservation and flow inside the rim}

Using the notations shown in fig. 7 , the mass conservation imposes:

$$
\Gamma(x+L(\psi) \cos \psi)=S(\psi) U(\psi)
$$

where $L(\psi)$ is the width of the $\operatorname{rim}, S(\psi)$ is the section of the rim and $U(\psi)$ is the mean velocity in the rim at a position along the contact line labelled by the angle $\psi$ (see fig. 7). As the width of the rim is of order of the capillary length it can be neglected with respect to $\mathrm{x}$ far from the apex. Near the apex $\psi$ is close to $\pi / 2$ making $L \cos \psi$ close to zero. Then, it appears to be reasonable to use a simplified form of the mass conservation:

$$
\Gamma x=S(\psi) U(\psi)
$$


(a)
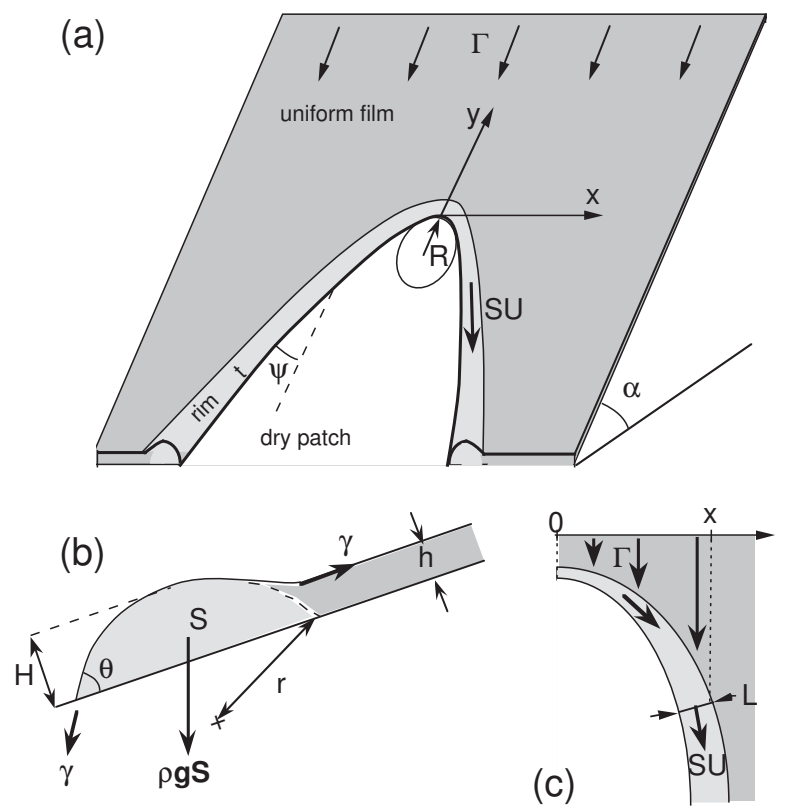

FIG. 7: Notations used in our models. (a): schematic of the flow, ( b): section of the liquid rim surrounding the dry patch and (c): sketch detailing the mass conservation for the rim.

Assuming a Stokes flow driven by gravity in the rim [13] gives the following expression for the mean liquid velocity $U(\psi)$ :

$$
\begin{aligned}
U(\psi) & =m f_{1}(\theta) \frac{U_{c} S(\psi)}{l_{c}^{2}} \sin \alpha \cos \psi \\
f_{1}(\theta) & =\frac{(1-\cos \theta)^{2}}{\theta-\sin \theta \cos \theta}
\end{aligned}
$$

where $U_{c}=\gamma / \eta$ and $l_{c}=(\gamma / \rho g)^{1 / 2}$ are respectively the capillary velocity and the capillary length and $m$ is a numerical factor taking into account the detail of the flow inside the rim. The exact value of $m$ depends on the shape of the rim and in particular on the contact angle $\theta$. Podgorski et al. [13] found a good estimate of $m$ by assuming the flow in the rim to be close to the lubrication flow inside a rivulet which gives $m=0.23$ for $\theta_{a}=52^{\circ}$. This estimate of $m$ varies very slowly with the contact angle and remains always between 0.23 and 0.24 in our experimental conditions. In the following, we will consider that $m$ is known and equal to $m=0.23$. 


\section{Balance of forces acting on the rim normally to the contact line}

In comparison to [13], we improve the description of the mechanical equilibrium of the rim by taking into account capillary effects linked to the radius of curvature $R$, hydrostatic pressure and momentum carried by the film. Let us first see in details the physical effects taken into account. We consider a small part of the rim (see fig. 8). Its volume is $S(\psi)(d s+$ $\left.d s^{\prime}\right) / 2=S(\psi) d s(1-L(\psi) /(2 R(\psi))$. Its weight projected perpendicularly to the contact line reads:

$$
d W=\rho g \sin \alpha \sin \psi d s\left(1-\frac{L(\psi)}{2 R(\psi)}\right)
$$

where $\mathrm{L}(\psi)$ is the width of the rim. This term contains a correction linked to the contact line curvature in comparison to previous initial modeling [13]. This correction comes directly from choosing the contact line as reference instead of using the rim central line. Using the relations $L(\psi)=2 r(\psi) \sin \theta$ and $S(\psi)=r(\psi)^{2}(\theta-\sin \theta \cos \theta)$, one obtains:

$$
d W=\rho g \sin \alpha \sin \psi d s\left(1-\frac{S(\psi)^{1 / 2} \sin \theta}{R(\psi)(\theta-\sin \theta \cos \theta)^{1 / 2}}\right)
$$

We consider now the capillary effects more directly linked to contact line curvature $R$. Theses ones can be reduced to an effective line tension that contains three contributions suggested on fig. 8. First (see fig. 8(a)) the element $d s$ is larger than $d s^{\prime}$ resulting in a force which tends to "open" the dry zone :

$$
d F_{1}=\gamma\left(d s-d s^{\prime} \cos \theta\right)
$$

Also, surface tension "pulls" along the contact line at the two segments boundaries (fig. 8(b)). Projection of these two forces of intensity $\gamma r(\psi) \theta$ on the normal at the contact line results in a force directed downwards :

$$
d F_{2}=2 \gamma r(\psi) \theta d \psi
$$

where $r(\psi)$ is the curvature at the rim cross section. Finally, the strong curvature of the rim cross section is associated to a capillary pressure $p \sim \gamma / r$ inside the rim. This change of pressure integrated on the rim cross section reduces the line tension (fig. 8(c)) and induces a third contribution acting in the opposite direction :

$$
d F_{3}=\frac{\gamma}{r(\psi)} S d \psi=\gamma r(\psi)(\theta-\sin \theta \cos \theta) d \psi
$$




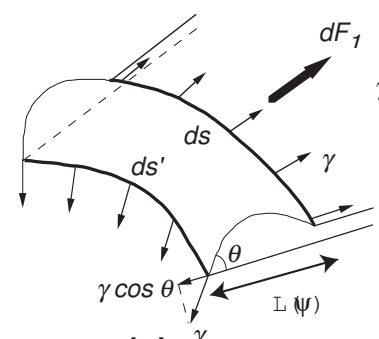

(a)

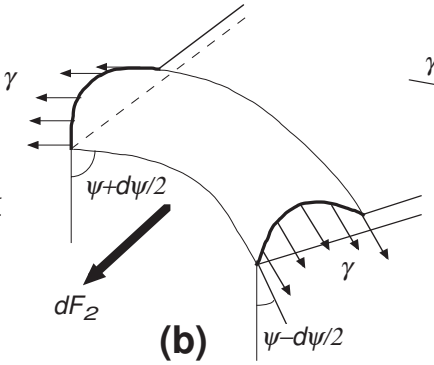

(b)

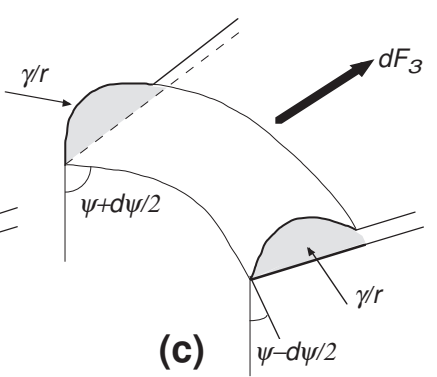

(c)

FIG. 8: Capillary effects and their resulting forces $d F_{1}, d F_{2}$ et $d F_{3}$ on a segment of the rim.

Using the relation $d s=R(\psi) d \psi$, one can write the capillary effects as

$$
d F_{c}=-d F_{1}+d F_{2}-d F_{3}=-\gamma d s(1-\cos \theta)+\gamma \frac{S(\psi)^{1 / 2}}{R(\psi)}(\theta-\sin \theta \cos \theta)^{1 / 2} d s
$$

As one can see, for both the weight and the capillary effects, the curvature of the contact line $R(\psi)$ induces corrections proportional to $S(\psi)^{1 / 2} / R(\psi)$. As $S(\psi)^{1 / 2} \simeq l_{c}$ these terms will induce a change in the shape only for value of $R(\psi)$ of order of $l_{c}$ which corresponds to the experimental loss of stability $R_{c} \simeq 3 l_{c}$. It is therefore expected that such effects should play a specific role in the stability issue. Podgorski et al.[15] took into account these corrective terms in their model, which leads to their prediction of the critical flow rate (4) but we here add more contributions that they have neglected.

First the hydrostatic pressure in the film results in a downwards force $d F_{h}$ acting on a segment $d s$ of the rim:

$$
d F_{h}=\rho g \cos \alpha\left(\frac{h^{2}}{2}\right) d s
$$

which can be rewritten using the relationship $\Gamma=\rho g \sin (\alpha) h^{3} /(3 \eta)$

$$
d F_{h}=\frac{\rho g}{2} \cos \alpha\left(\frac{3 \eta \Gamma}{\rho g \sin \alpha}\right)^{2 / 3} d s
$$

This term is negligible for large plate slope $\alpha$ but becomes dominant at small $\alpha$. If one looks to orders of magnitude, the rim weight and the hydrostatic pressure will be of the same order when:

$$
\rho g S \sin \alpha \simeq \rho g \cos \alpha \frac{h^{2}}{2} d s
$$

which leads to a value of $\alpha$ of order of $2^{o}$. Although this term is almost always negligible, it will prevent the divergence for small plate slope when $\alpha<2^{o}$ where it will become dominant 
with respect to $\mathrm{dW}$.

The momentum carried by the film leads to another effective force $d F_{i}$. According to [20] this force can be written as :

$$
d F_{i}=\rho \frac{2}{15} \frac{g^{2} \sin ^{2}(\alpha) h^{5}}{\nu^{2}} \sin ^{2} \psi d s
$$

which can be written as

$$
d F_{i}=\frac{2}{15} \frac{\rho^{3} g^{2} \sin ^{2} \alpha \sin ^{2} \psi}{\eta^{2}}\left(\frac{3 \eta \Gamma}{\rho g \sin \alpha}\right)^{5 / 3} d s
$$

As these additional terms are only first (or larger) order corrections, they have only a small influence on the shape of the dry patch. But they can clearly have an influence on its stability. Using these additional terms the mechanical equilibrium is now given by:

$$
d W+d F_{c}+d F_{h}+d F_{i}=0
$$

which leads to

$$
\begin{aligned}
\gamma(1-\cos \theta) & =\rho g S(\psi) \sin \alpha \sin \psi\left(1-\frac{S^{1 / 2}}{R(\psi)} \frac{\sin \theta}{(\theta-\sin \theta \cos \theta)^{1 / 2}}\right) \\
& +\gamma \frac{S^{1 / 2}}{R(\psi)}(\theta-\sin \theta \cos \theta)^{1 / 2}+\frac{\rho g}{2} \cos \alpha\left(\frac{3 \eta \Gamma}{\rho g \sin \alpha}\right)^{2 / 3} \\
& +\frac{2}{15} \frac{\rho^{3} g^{2} \sin ^{2} \alpha \sin ^{2} \psi}{\eta^{2}}\left(\frac{3 \eta \Gamma}{\rho g \sin \alpha}\right)^{5 / 3}
\end{aligned}
$$

\section{Evolution of the radius of curvature at the apex}

Combining eq. (7) and eq. (8) near the apex $(\psi \simeq \pi / 2, \cos \psi \simeq x / R$ and $\sin \psi \simeq 1)$, one can find an expression for the section $S(\psi)$ :

$$
S(\psi)=\left(\frac{l_{c}^{2} \Gamma R(\psi)}{U_{c} m f_{1}(\theta) \sin \alpha}\right)^{1 / 2}\left(\psi \simeq \frac{\pi}{2}\right)
$$

Using the dimensionless radius of curvature at the apex $R^{*}=R / l_{c}$ and flow rate $\Gamma^{*}=$ $\Gamma /\left(U_{c} l_{c}\right),(21)$ becomes now:

$$
\begin{aligned}
1 & =f_{3}(\theta) R^{* 1 / 2}\left(\Gamma^{*} \sin \alpha\right)^{1 / 2}-\frac{\sin \theta(1-\cos \theta)^{1 / 2}}{(\theta-\sin \theta \cos \theta)^{1 / 2}} f_{3}(\theta)^{3 / 2} \Gamma^{* 3 / 4} R^{*-1 / 4} \sin ^{1 / 4} \alpha \\
& +\frac{(\theta-\sin \theta \cos \theta)^{1 / 2}}{\sin ^{1 / 4} \alpha(1-\cos \theta)^{1 / 2}} f_{3}(\theta)^{1 / 2} R^{*-3 / 4} \Gamma^{* 1 / 4} \\
& +\frac{3^{2 / 3}}{1-\cos \theta}\left(\frac{\cos \alpha}{2}\left(\frac{\Gamma^{*}}{\sin \alpha}\right)^{2 / 3}+\frac{2}{5} \frac{\rho^{1 / 2} \gamma^{3 / 2}}{\eta^{2} g^{1 / 2}}\left(\frac{\Gamma^{*}}{\sin \alpha}\right)^{5 / 3}\right)
\end{aligned}
$$




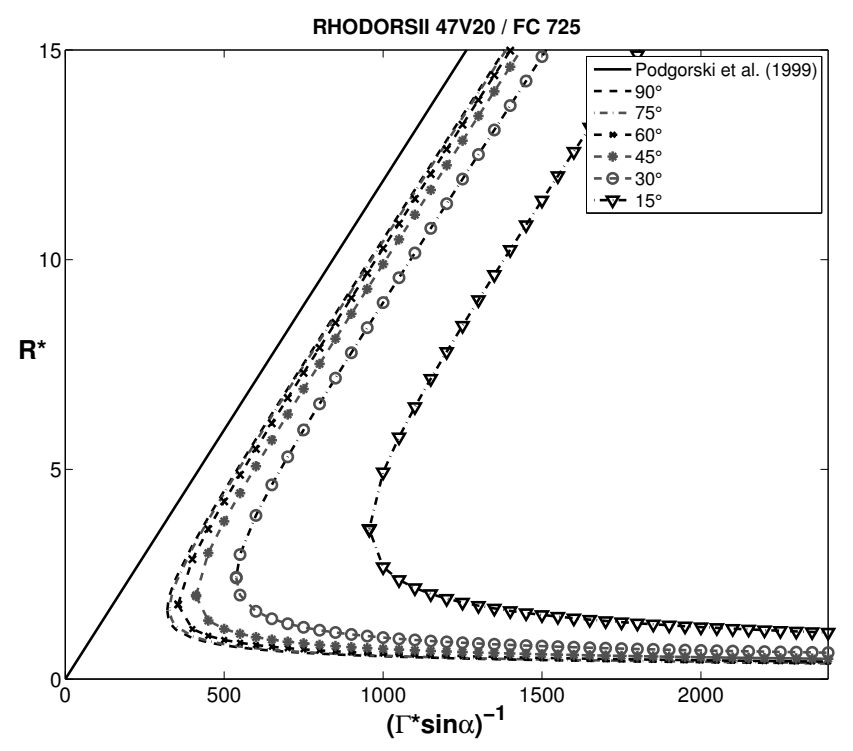

FIG. 9: Improved model 1 for the evolution of the radius of curvature at the apex with the flow rate $\left(m=0.23\right.$ and $\left.\theta=52^{\circ}\right)$.

where $f_{3}(\theta)$ is the function

$$
f_{3}(\theta)=\frac{(\theta-\sin \theta \cos \theta)^{1 / 2}}{m^{1 / 2}(1-\cos \theta)^{2}} .
$$

This equation gives an implicit relation between $R^{*}$ and $\Gamma^{*}$ and its numerical resolution can be seen on fig. 9. As one can see, this model predicts two possible solutions at the same flow rate $\Gamma^{*}$. One of these solutions (lower branch) only exists for radius of curvature smaller than the critical value observed experimentally $\left(R^{*}<R_{c}^{*} \simeq 3\right)$. A linear stability analysis presented thereafter will show that this branch is unstable with respect to small perturbation in $R$. The other (upper branch) appears to be almost linear (with $(\Gamma \sin \alpha)^{-1}$ ) for sufficiently high value of $R^{*}$. The observed slope is the same as the one obtained by Podgorski et al. [13] but combined with a vertical shift of the curve that depends on plate slope. All these features are consistent with [15] and with what we have seen on the experiment.

A quantitative comparison between this improved model and our experimental data is presented in figs. 10, 11 and 12 for the evolution of the radius of curvature at different plate slopes. As one can see a quite good agreement for high plate inclination is found for 47V20 oil and for $\alpha>45^{\circ}$. For Huber oil the agreement is only good for an inclination angle higher 

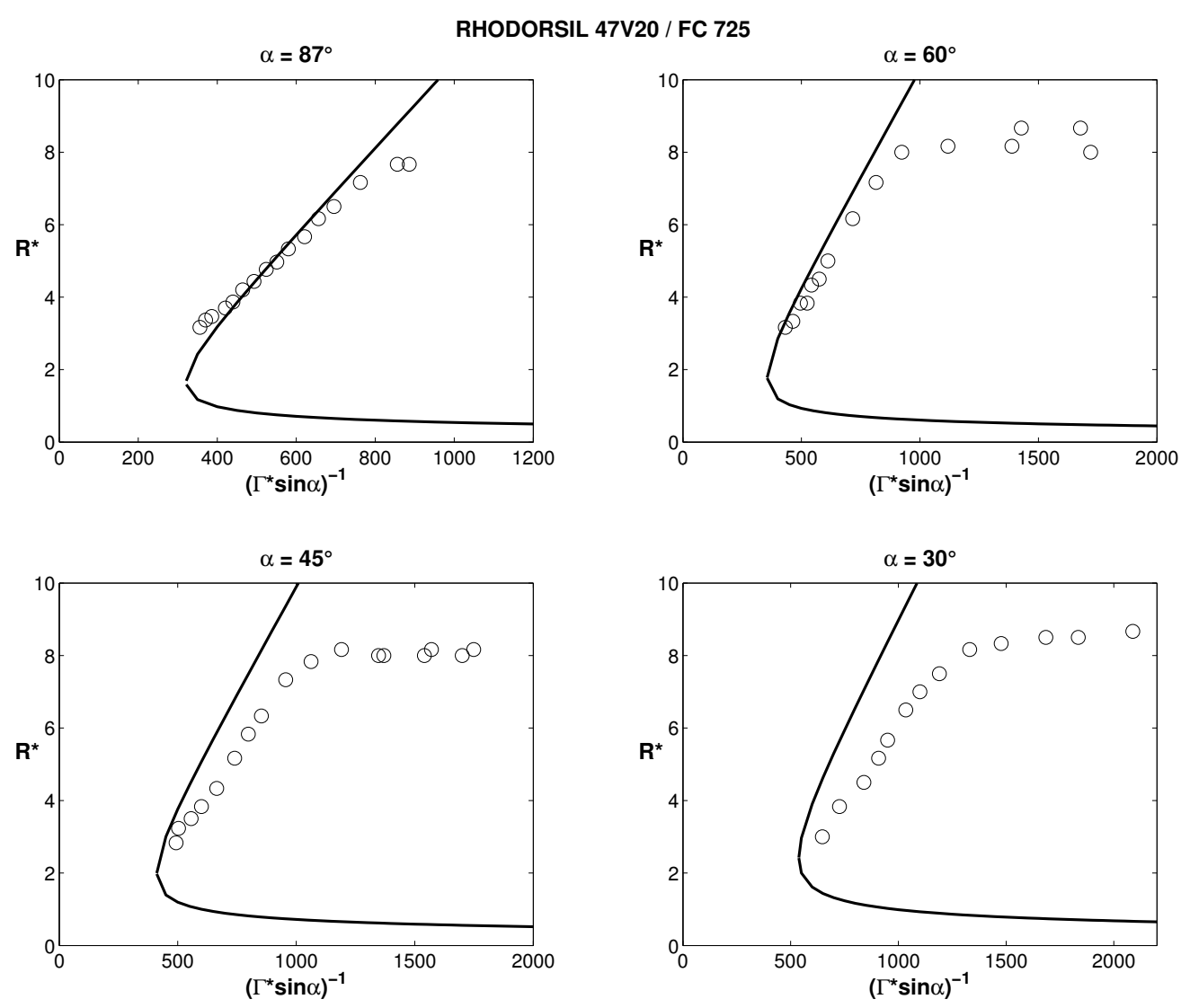

FIG. 10: Theory (model 1) and experiments for the Rhodorsil 47V20 oil $\left(m=0.23, \eta=1.76 .10^{-2}\right.$ Pa.s and $\left.\theta=52^{\circ}\right)$. This model is in good agreement for high plate slope $\left(\alpha>45^{\circ}\right)$ but presents discrepancies for low plate slope.

than $65^{\circ}$. One can also remark that the prediction of the shift between the curves is quite good for the $20 \mathrm{cP}$ oil (Rhodorsil 47V20) and of the good order of magnitude for the $10 \mathrm{cP}$ oil (HUBER). For the $5 \mathrm{cP}$ oil (Rhodorsil 47V5), the agreement is only qualitative for a vertical plate and becomes worse and worse as the plate slope decreases. As one can see, all oils exhibit discrepancies for small inclination angle. These discrepancies might be linked to the modeling of the flow inside the rim, which is assumed to be a Stokes flow driven by gravity. This assumption becomes worse and worse as the inclination angle becomes small (for small inclination angle one can expect the flow to be driven by hydrostatic pressure). One can also observe than discrepancies also appear for larger inclination angle when one decreases the liquid viscosity. These discrepancies are certainly linked to inertial effect in the flow in- 

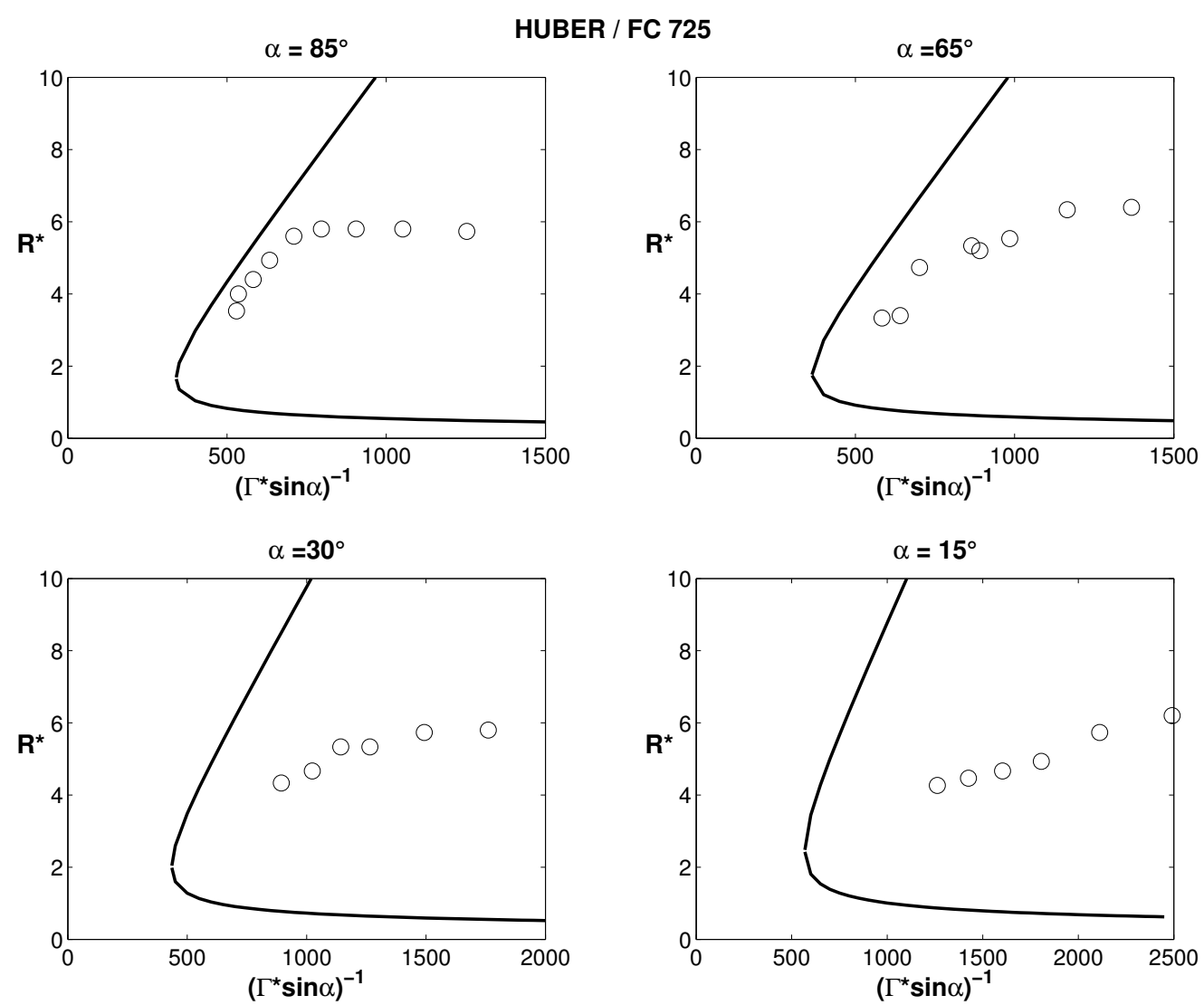

FIG. 11: Theory (model 1$)$ and experiments for Huber oil $\left(m=0.23, \eta=1.155 .10^{-2}\right.$ Pa.s and $\theta=$ $52^{\circ}$ ). This model is in good agreement for plate slope $\alpha>65^{\circ}$ but the agreement becomes worse and worse as the plate slope decreases.

side the rim. The effect of inertia in the flow inside the rim will be studied in the next section.

\section{E. Stability of the two branches}

One can remark on fig. 9 that the solutions provided by this approach are reminiscent of a saddle-node bifurcation. In analogy with this situation, and as the lower branch of the solution is never observed experimentally, we can expect the dry patch to become unstable for sufficiently low radius of curvature i.e. for this lower branch. To check this, we now study the stability of the improved model solution with respect to a small variation of the radius of curvature with constant flow rate and plate slope (procedure illustrated on 

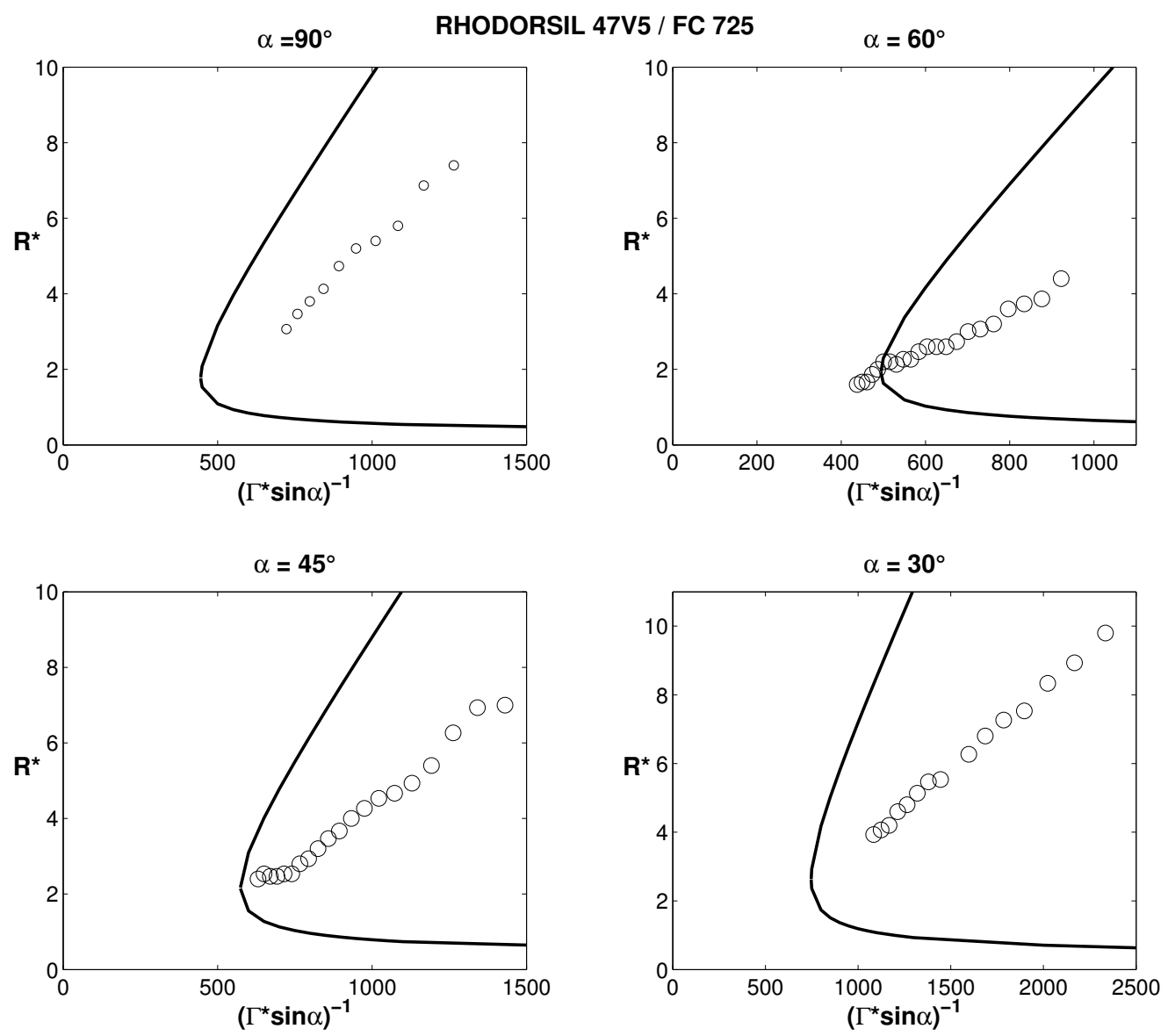

FIG. 12: Theory (model 1) and experiments for the Rhodorsil 47V5 oil $\left(m=0.23, \eta=4.6 .10^{-2}\right.$ Pa.s and $\theta=52^{\circ}$ ). This model presents only qualitative agreement for almost vertical plate and becomes worse and worse as the plate slope decreases.

fig. 13). Let us consider an out of equilibrium dry patch shape. Equation(23) must then be rewritten in terms of a non-dimensional force $\tilde{F}$ that tends to open the dry zone when positive, or to close it when negative:

$$
\begin{aligned}
\tilde{F} & =1-f_{3}(\theta) R^{* 1 / 2}\left(\Gamma^{*} \sin \alpha\right)^{1 / 2}+\frac{\sin \theta}{(1-\cos \theta)^{1 / 2}}(\theta-\sin \theta \cos \theta)^{1 / 2} f_{3}(\theta)^{3 / 2} \Gamma^{* 3 / 4} R^{*-1 / 4} \sin ^{1 / 4}(\alpha) \\
& -\frac{(\theta-\sin \theta \cos \theta)^{1 / 2}}{\sin ^{1 / 4}(\alpha)(1-\cos \theta)^{1 / 2}} f_{3}(\theta)^{1 / 2} R^{*-3 / 4} \Gamma^{* 1 / 4} \\
& -\frac{3^{2 / 3}}{1-\cos \theta}\left(\cos \alpha\left(\frac{\Gamma^{*}}{\sin \alpha}\right)^{2 / 3}+\frac{2}{5} \frac{\rho^{1 / 2} \gamma^{3 / 2}}{\eta^{2} g^{1 / 2}}\left(\frac{\Gamma^{*}}{\sin \alpha}\right)^{5 / 3}\right)
\end{aligned}
$$




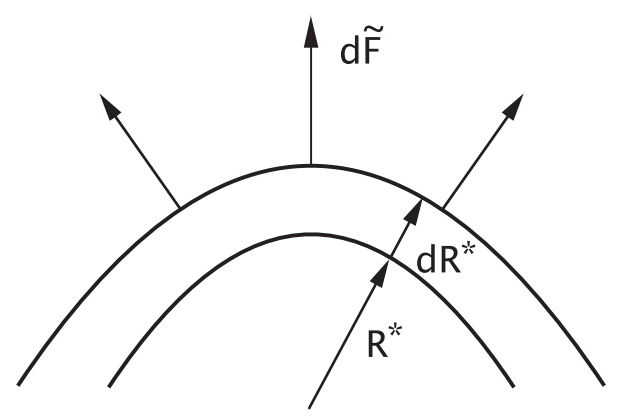

FIG. 13: Illustration of the procedure used for the linear stability analysis

and its variation upon the radius of curvature reads:

$$
\begin{aligned}
\frac{d \tilde{F}}{d R^{*}} & =-\frac{1}{2} f_{3}(\theta) R^{*-1 / 2}\left(\Gamma^{*} \sin \alpha\right)^{1 / 2}-\frac{1}{4} \frac{\sin \theta(1-\cos \theta)^{1 / 2}}{(\theta-\sin \theta \cos \theta)^{1 / 2}} f_{3}(\theta)^{3 / 2} \Gamma^{* 3 / 4} R^{*-5 / 4} \sin ^{1 / 4}(\alpha) \\
& +\frac{3}{4} \frac{(\theta-\sin \theta \cos \theta)^{1 / 2}}{\sin ^{1 / 4}(\alpha)(1-\cos \theta)^{1 / 2}} f_{3}(\theta)^{1 / 2} R^{*-7 / 4} \Gamma^{* 1 / 4}
\end{aligned}
$$

A small increase of the radius of curvature $d R^{*}$ corresponds to a slight opening of the dry patch, which leads to a non compensated force $d \tilde{F}$. Hence, the dry patch will be stable if the force $d \tilde{F}$ tends to close it up. This gives the following condition of stability:

$$
\frac{d \tilde{F}}{d R^{*}}<0
$$

If one looks to the sign of the different contributions, one can conclude that the rim weight is stabilizing and the capillary effects linked to the curvature of the contact line are on the contrary destabilizing. As one can remark, the condition of stability is independent of both the hydrostatic pressure term and of the film momentum contribution, that do not depend on $R^{*}$. The implicit equation $d \tilde{F} / d R^{*}=0$ gives the limit of stability of the improved model solution and can be solved numerically for each plate slope $\alpha$. An example is presented in fig. 14. As expected, the solution corresponding to the lower branch is unstable which could explain the fact we never observe it in experiments.

\section{F. Predicting rewetting: the critical flow rate}

We now focus on the question of the critical flow rate above which the dry patch disappears. As one can see on fig. 9, for each plate slope $\alpha$, this model predicts the existence of a critical flow rate $\Gamma_{c}$ above which there is no solution for the dry patch shape. This was yet 


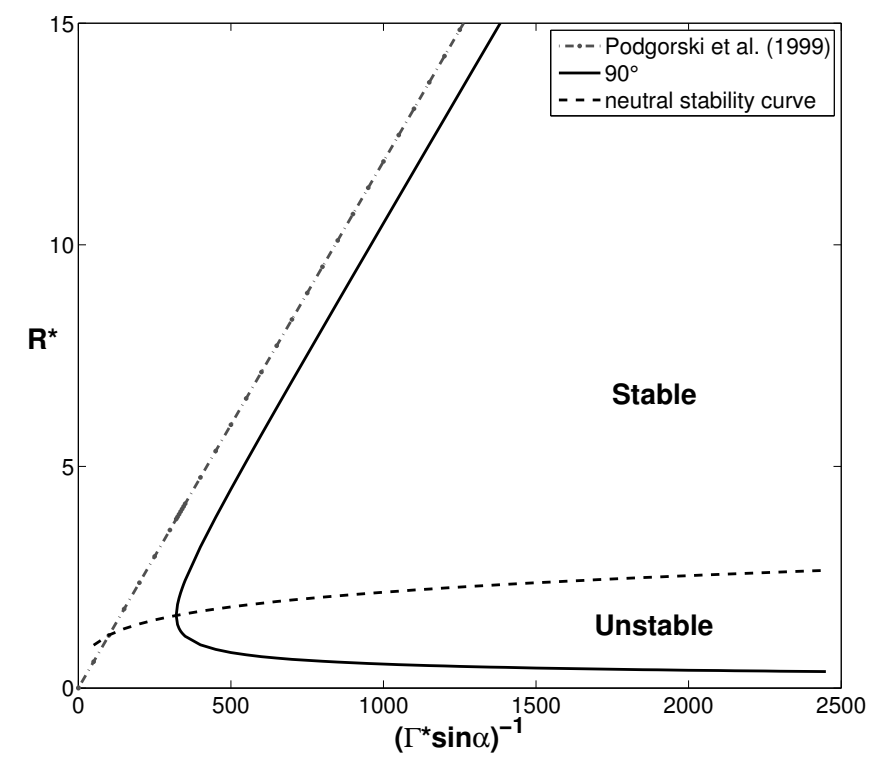

FIG. 14: Improved model 1 and limit of stability when $\alpha=90^{\circ}$ for Rhodorsil $47 \mathrm{~V} 20$ oil $(\mathrm{m}=0.23$ and $\left.\theta=52^{\circ}\right)$

obtained in the simplified approach of Podgorski et al. [15] and comes mainly from the fact that we have taken into account curvature terms of the contact line. This very general idea goes back to S.D.R Wilson [20], who however did not realize the existence of two branches, as evidenced by figs. 9 and 18. Another important point, evidenced by these figures, is that these curvature terms lead to a critical flow rate reached when the curvature at the apex is close to three times the capillary length, an observation yet used by Rio et al. [18] to build approximates of $\Gamma_{c}$. The present modeling allows us to get a much better prediction for $\Gamma_{c}$ by identifying its value to the one of the "turning point" in fig. 9, where the two branches are meeting. No dry patch can survive above this value that is given by the equations:

$$
\begin{aligned}
\tilde{F} & =0 \\
\frac{d \tilde{F}}{d R^{*}} & =0
\end{aligned}
$$

in which $\tilde{F}$ and $d \tilde{F} / d R^{*}$ are given respectively by eq. (25) and eq. (26). We have solved numerically this system of equations which has allowed us to obtained the plots of $\Gamma_{c}(\alpha)$ (given in fig. 15) expected for the three oils used in this study.

A more realistic prediction of $\Gamma_{c}$ than in [15] appears on these graphs. Instead of an un- 
physical divergence in the limit $\alpha=0$, one obtains a vanishing $\Gamma_{c}$ that corresponds to the small slope limit discussed in the first paper from Podgorski [13]. As in this reference, this behavior is linked to the hydrostatic pressure term taken into account in the flowing film. At large plate slope, a decrease of $\Gamma_{c}$ is observed, in qualitative agreement with our data plotted in fig. 15. The crossover between these two regimes (small and large plate slope) leads to a maximum for $\Gamma_{c}$ that seems to depend only weakly on the viscosity and is close to $\alpha=10^{\circ}$ for the three oils. This plate inclination corresponds to the most difficult situation for plate wetting, the best situations being obtained in the limits $\alpha=0^{\circ}$ and $\alpha=90^{\circ}$.

The comparison with our data is satisfactory for the $20 \mathrm{cP}$ oil, but not so much for the two other oils, though the model indicates the right tendencies and the right order of magnitude. If we consider the values observed for $\alpha=90^{\circ}, \Gamma_{c}$ is overestimated of nearly $50 \%$ for the 5 $\mathrm{cP}$ oil, $25 \%$ for the $10 \mathrm{cP}$ oil and only $5-10 \%$ for the $20 \mathrm{cP}$ oil. These results are not so bad considering the difficulty of the problem and the fact that our model does not contain any adjustable parameter, except perhaps the value of the parameter $\mathrm{m}$ that does not however vary a lot here $(\mathrm{m}$ is fixed to the lubrication limit here $\mathrm{m}=0.23)$. These discrepancies are however sufficient to motivate the next part of this paper, where we try to refine the description of the flow inside the rim, using the ideas previously imagined by S.D.R Wilson [20] but slightly modified with respect to boundary layer effects. We also improve his approach by calculating explicitly the shape of the dry patch while in [20] it was approximated by a parabola. 

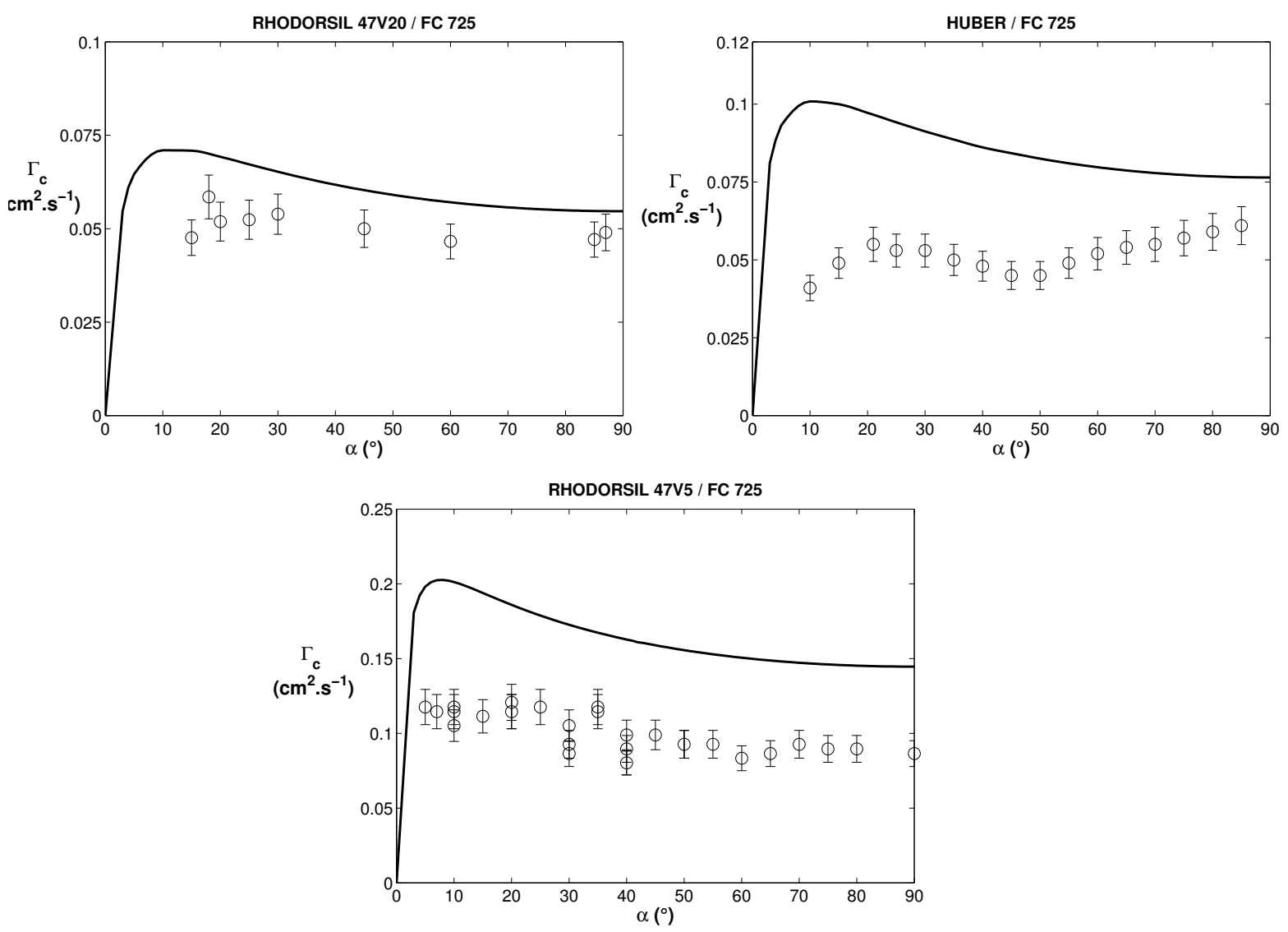

FIG. 15: Comparisons of the measured critical flow rate to the predictions of eq. (28).

\section{SECOND STEP : IMPROVING THE DESCRIPTION OF THE FLOW INSIDE THE RIM (MODEL 2)}

As the momentum carried by the film appears to have a non negligible effect on the prediction of the critical flow rate, one can expect that inertia should also modify the flow structure inside the rim. In order to take into account such an effect, we consider a segment of rim of length $d s$ as illustrated on fig.16:

- at the coordinate $s$, the momentum flux entering the rim segment is $\left[\rho S(\psi) \bar{U}^{2}\right]_{s}$

- at the coordinate $s+d s$, the momentum fluw leaving the rim segment is $\left[\rho S(\psi) \bar{U}^{2}\right]_{s+d s}$

- All along $d s$, the film provides the rim with a momentum flux $\rho\left(h V^{2}\right) \cos \psi d x$

This variation of the momentum flux must be balanced by the gravity acceleration $\rho g S(\psi) \sin \alpha \cos \psi d s$ and by the viscous friction in the boundary layer formed near the glass 


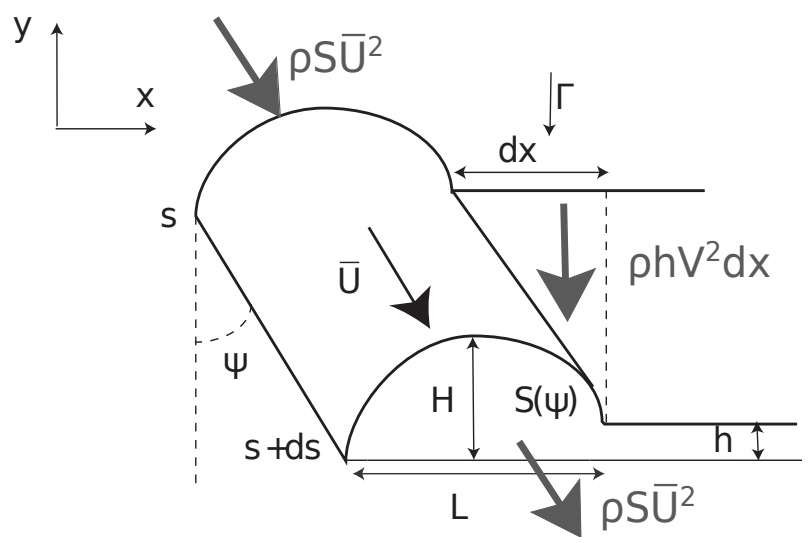

FIG. 16: Notations used in our analysis of the flow inside the rim

plate. If we assumed a fully developed boundary layer, this viscous friction is given by $\beta \eta \bar{U} L / H$ where $\beta$ is a form factor. Using $d x=d s \sin \psi$, we obtain:

$$
-\frac{d}{d s}\left[\rho S(\psi) \bar{U}^{2}\right]+\rho\left(h V^{2}\right) \cos \psi \sin \psi+\rho g S(\psi) \sin \alpha \cos \psi-\beta \eta \frac{\bar{U} L}{H}=0
$$

This relation can be rewritten in the form :

$$
\frac{d \bar{U}}{d s}(S(\psi) \bar{U})+\frac{d(S(\psi) \bar{U})}{d s} \bar{U}+\beta \nu \frac{\bar{U} L}{H}=\left(h V^{2}\right) \cos \psi \sin \psi+g S(\psi) \sin \alpha \cos \psi
$$

The derivation of (7) with respect to $s$ yields to:

$$
\frac{d(S(\psi) \bar{U})}{d s}=\Gamma \sin \psi
$$

As we work near the apex of the dry patch, one can expect the variation of the section to be negligible with respect to the variation of the mean velocity [20] which leads to:

$$
\frac{1}{S(\psi)} \frac{d S(\psi)}{d s}<<\frac{1}{\bar{U}} \frac{d \bar{U}}{d s}
$$

And then one can find

$$
\frac{d \bar{U}}{d s}=\frac{\Gamma \sin \psi}{S(\psi)}
$$

One can now easily rewrite eq. (30) as:

$$
2 \Gamma \sin (\psi) \bar{U}+\beta \nu \frac{\bar{U} L}{H}=\left(h V^{2}\right) \cos \psi \sin \psi+g S(\psi) \sin \alpha \cos \psi
$$

The form factor $\beta$ is determined in the limit of a pure Stokes flow inside the rim where the velocity is given by eq. (8) :

$$
\beta=\frac{H}{m f_{1}(\theta) L}
$$




\begin{tabular}{|l|c|c|}
\hline Oil & $\Gamma_{t}$ & $\Gamma_{c}$ \\
\hline Rhodorsil 47V20 & $1.11 \mathrm{~cm}^{2} . \mathrm{s}^{-1}$ & $0.05 \mathrm{~cm}^{2} \cdot \mathrm{s}^{-1}$ \\
\hline Huber & $0.5 \mathrm{~cm}^{2} . \mathrm{s}^{-1}$ & $0.05 \mathrm{~cm}^{2} . \mathrm{s}^{-1}$ \\
\hline Rhodorsil 47V5 & $0.3 \mathrm{~cm}^{2} . \mathrm{s}^{-1}$ & $0.1 \mathrm{~cm}^{2} . \mathrm{s}^{-1}$ \\
\hline
\end{tabular}

TABLE IV: Estimates of the flow rate $\Gamma_{t}$ where inertial effects are of the same order of magnitude than the viscous friction and comparison with the critical flow rate.

As the Froude Number is small $\left(F r \simeq 10^{-2}\right)$, we neglect the momentum carried by the film with respect to the rim weight and finally find:

$$
\bar{U}=\frac{m f_{1}(\theta) U_{c} g S(\psi) \sin \alpha}{l_{c}^{2}} \frac{\cos \psi}{1+2 m f_{1}(\theta) \frac{\Gamma}{\nu} \sin \psi}
$$

This expression is similar to eq. (8) moderated by a factor depending on the Reynolds number of the film $\Gamma / \nu$. To estimate the effect of inertia on the flow inside the rim, one can compute a typical flow rate $\Gamma_{t}$ for which viscous friction and inertia are equal. This is simply done by equating the two terms in the left part of eq. (34) and leads to:

$$
\Gamma_{t} \simeq \frac{\nu \sin \psi}{2 m f_{1}(\theta)}
$$

The corresponding flow rate $\Gamma_{t}$ for the three oils used in our study are given in Tab. IV. One can remark than this flow rate is quite higher than the critical flow rate for the Rhodorsil $47 \mathrm{~V} 20$ oil but of the same order of magnitude for the Huber and Rhodorsil 47V5 oil. One can then expect these inertial effects to play a non negligible role on stability for these two last oils and be negligible for the first one, in agreement with what we found in fig. 15 .

\section{A. Implication for the shape a dry patch}

To evaluate the effect on the shape prediction we first combine this improved description of the flow in the rim with the simple mechanical equilibrium of the rim used in Podgorski et al [13]. This simple form of the mechanical equilibrium will be used here to avoid too much algebra and will allow us to explain simply why the arch shape appears insensitive to inertial effects if one rescales the apex curvature in an appropriate way. Nevertheless in the following subsections where the evolution of the radius and curvature and the critical 
flow rate are more precisely modeled, we will use the improved version of the mechanical equilibrium i.e. eq. (21). Combining eqs. (7) and (36) with the first order balance of force at the contact line given by:

$$
\gamma(1-\cos \theta)=\rho g S(\psi) \sin \alpha \sin \psi
$$

yields a new prediction for the dry patch shape that generalizes that of Podgorki et al [13] to flows having both inertial and viscous effect incluced:

$$
\begin{aligned}
x & =R_{v} \frac{1}{1+g(\theta) \Gamma \sin \psi} \frac{\cos \psi}{\sin ^{2} \psi} \\
y & =R_{v}\left(\frac{1}{3}\left(\frac{1-3 \cos ^{2} \psi}{\sin ^{3} \psi}-1\right)+\frac{g(\theta) \Gamma}{2}\left(\frac{1}{\sin ^{2} \psi}-1\right)\right) \\
& +R_{v}\left(g^{3}(\theta) \Gamma^{3} \ln \left(\frac{(1+g(\theta) \Gamma \sin \psi}{1+g(\theta) \Gamma \sin \psi}\right)+(g(\theta) \Gamma-1)\left(\frac{1+g(\theta) \Gamma}{1+g(\theta) \Gamma \sin \psi}-1\right)\right)
\end{aligned}
$$

where

$$
\begin{aligned}
R_{v} & =m f_{2}(\theta) \frac{l_{c}^{2}}{\sin (\alpha)} \frac{U_{c}}{\Gamma} \\
g(\theta) & =\frac{2 m f_{1}(\theta)}{\nu} .
\end{aligned}
$$

Comparison between the shape predictions eqs. (2) and (39) are given in fig. 17 for the three oils at a flow rate close to the critical flow rate $\Gamma_{c}$. As one can see, even at these large flow rates, these inertial effects do not affect the shape of the dry patch. This very important finding explains why the shape of dry patches is so robust and -though calculated initially in the viscous limit- works even when viscous and inertial effects are combined.

\section{B. Evolution of the radius of curvature at the apex}

We explore now the evolution of the radius of curvature, with the completed, improved balance of forces at the contact line (21) combined with the mass conservation (7) and with the flow inside the rim calculated in (36). Using the dimensionless radius of curvature $R^{*}$ 

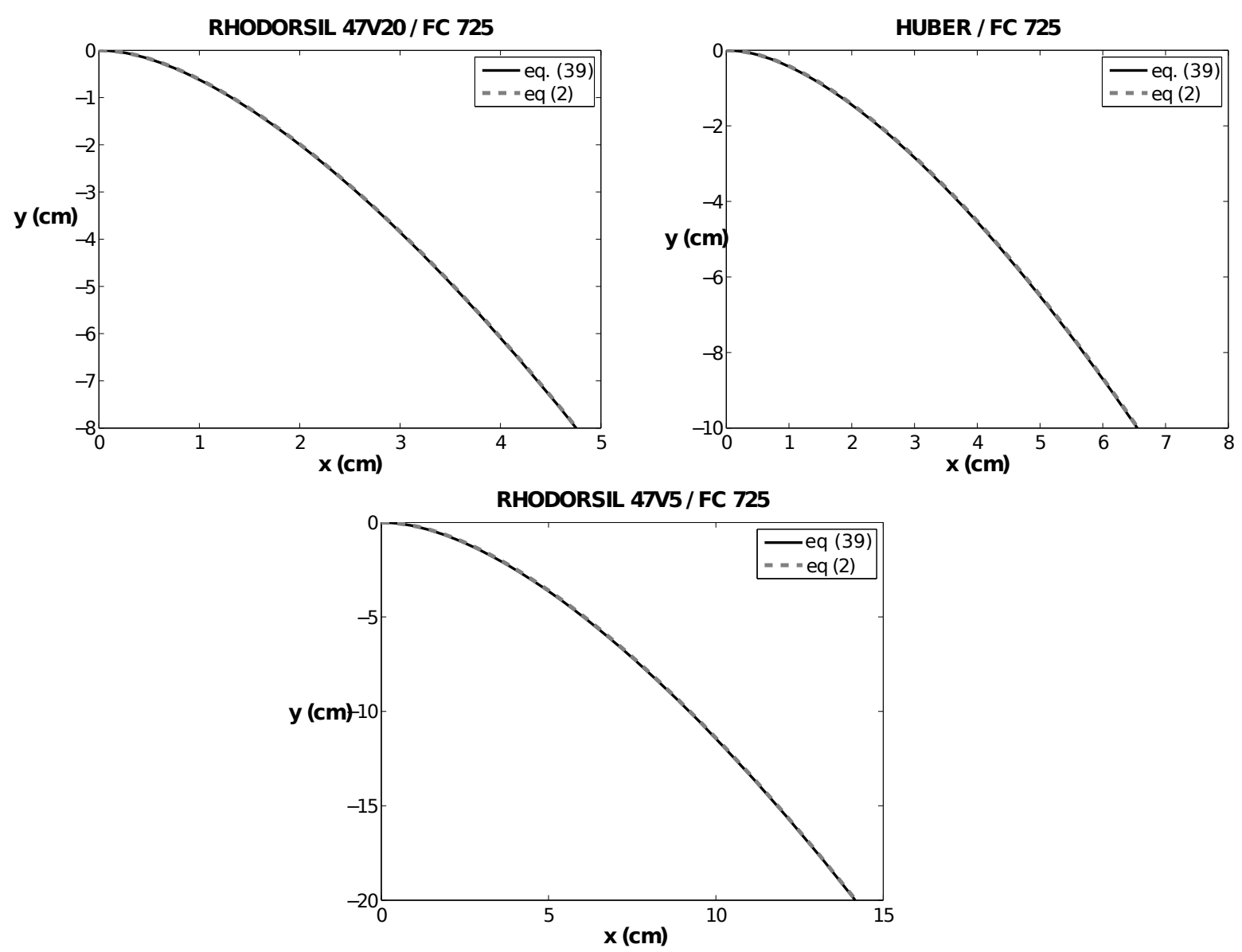

FIG. 17: Comparison between shape predictions eq. (2) in plain line and eq. (39) in dashed line for a flow rate $\Gamma=0.05 \mathrm{~cm}^{2} \cdot \mathrm{s}^{-1}$ and a plate slope $\alpha=90^{\circ}$.

and flow rate $\Gamma^{*},(21)$ becomes near the apex :

$$
\begin{aligned}
1 & =f_{3}(\theta) R^{* 1 / 2}\left(\Gamma^{*} \sin \alpha\right)^{1 / 2}\left(1+g^{*}(\theta) \Gamma^{*}\right)^{1 / 2} \\
& -\frac{\sin \theta(1-\cos \theta)^{1 / 2}}{(\theta-\sin \theta \cos \theta)^{1 / 2}} f_{3}(\theta)^{3 / 2} \Gamma^{* 3 / 4} R^{*-1 / 4}\left(1+g^{*}(\theta) \Gamma^{*}\right)^{3 / 4} \sin ^{1 / 4}(\alpha) \\
& +\frac{(\theta-\sin \theta \cos \theta)^{1 / 2}}{(1-\cos \theta)^{1 / 2} \sin ^{1 / 4}(\alpha)} f_{3}(\theta)^{1 / 2} R^{*-3 / 4} \Gamma^{* 1 / 4}\left(1+g^{*}(\theta) \Gamma^{*}\right)^{1 / 4} \\
& +\frac{3^{2 / 3}}{1-\cos \theta}\left(\frac{\cos \alpha}{2}\left(\frac{\Gamma^{*}}{\sin \alpha}\right)^{2 / 3}+\frac{2}{5} \frac{\rho^{1 / 2} \gamma^{3 / 2}}{\eta^{2} g^{1 / 2}} \Gamma^{* 5 / 3} \sin ^{1 / 3}(\alpha)\right)
\end{aligned}
$$

where

$$
g^{*}(\theta)=\frac{2 m f_{1}(\theta) U_{c} l_{c}}{\nu}
$$

Resolutions of (41) are given in fig. 18. As one can see, the solutions are of similar structure to those of (23) and a similar analysis of stability confirms that the lower branch is unstable. 

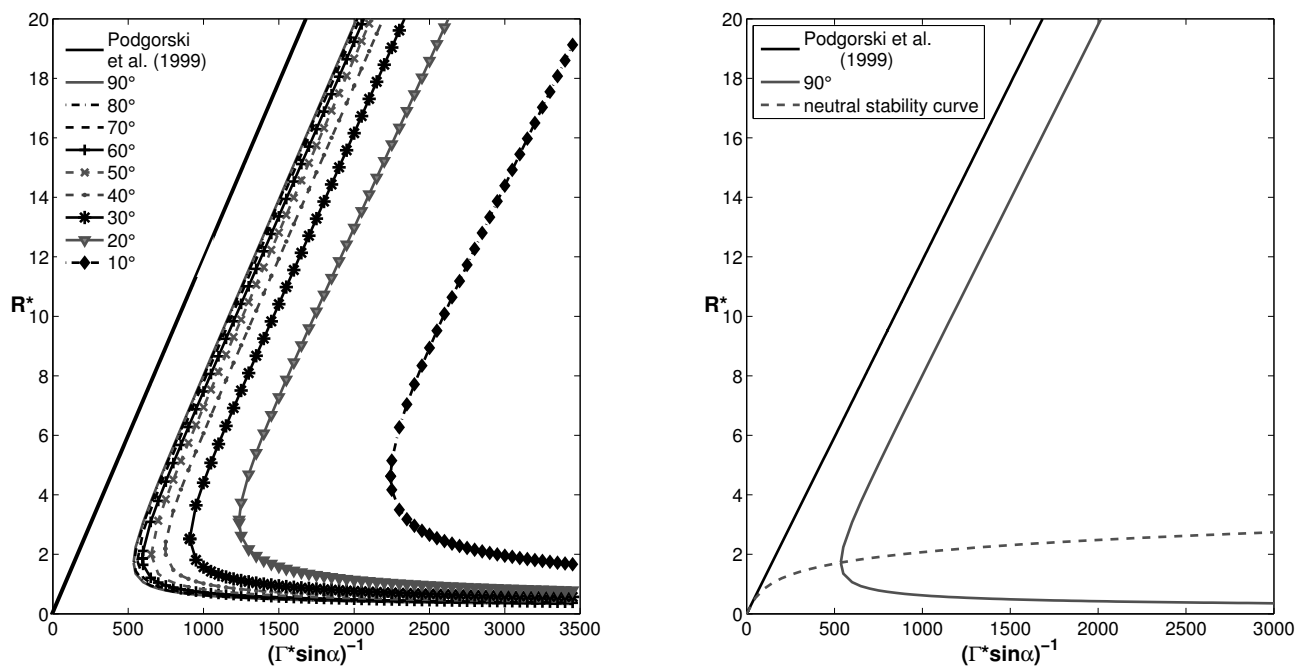

FIG. 18: Improved predictions of model 2 for the evolution of the radius of curvature at the apex with flow rate $\left(m=0.23\right.$ and $\left.\theta=52^{\circ}\right)$ and stability limit.

Comparisons of this new prediction with the experiments are given fig. 19, 20 and 21 for respectively the Rhodorsil 47V20, Huber and Rhodorsil 47V5 oils. It appears that, as expected, inertial effects inside the rim do not change significantly the prediction for the most viscous oils (Rhodorsil 47V20 and Huber oils), but improve clearly the prediction for the less viscous oil. As previously, the agreement is quite good for the Rhodorsil 47V20 oil for plate slope larger than $45^{\circ}$. The agreement is only good for the Huber oil for plate slope larger than $65^{\circ}$ and is just qualitative for the Rhodorsil $47 \mathrm{~V} 5$ oil for vertical plate. As in the previous section, the discrepancies observed for small inclination angles might be linked to the modeling of the viscous part of the flow, which is supposed to be driven by gravity. For high inclination angles and for the less viscous oils (Huber and Rhodorsil 47V5 oils), the discrepancies between the model and the experiment are probably linked to the apparition of long (Kapitza) waves on the liquid film (see fig. 22). Using the famous Benjamin criterion [26] one can easily compute the flow rate $\Gamma_{w}$ corresponding to the appereance of these long waves:

$$
\Gamma_{w}=\frac{5 \nu \cos \alpha}{6}
$$

Typical values of the flow rate $\Gamma_{w}$ and of the corresponding non dimensional parameter $\left(\Gamma_{w}^{*} \sin \alpha\right)^{-1}$ are given in Table $\mathrm{V}$. As one can see these flow rate are in the typical range of 


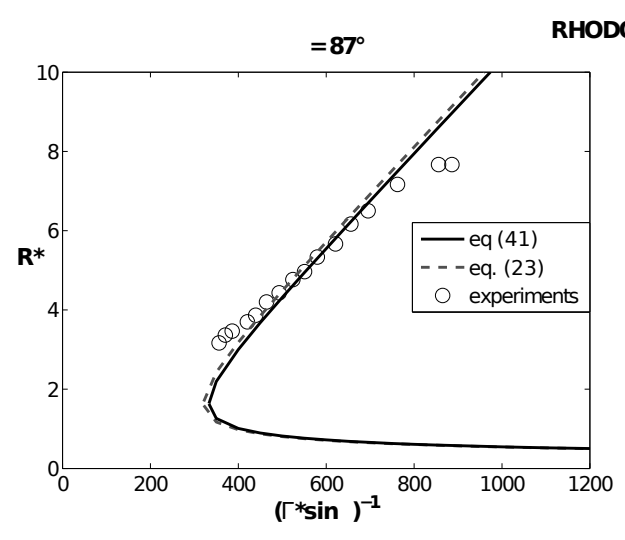

725 4720 / FC

725
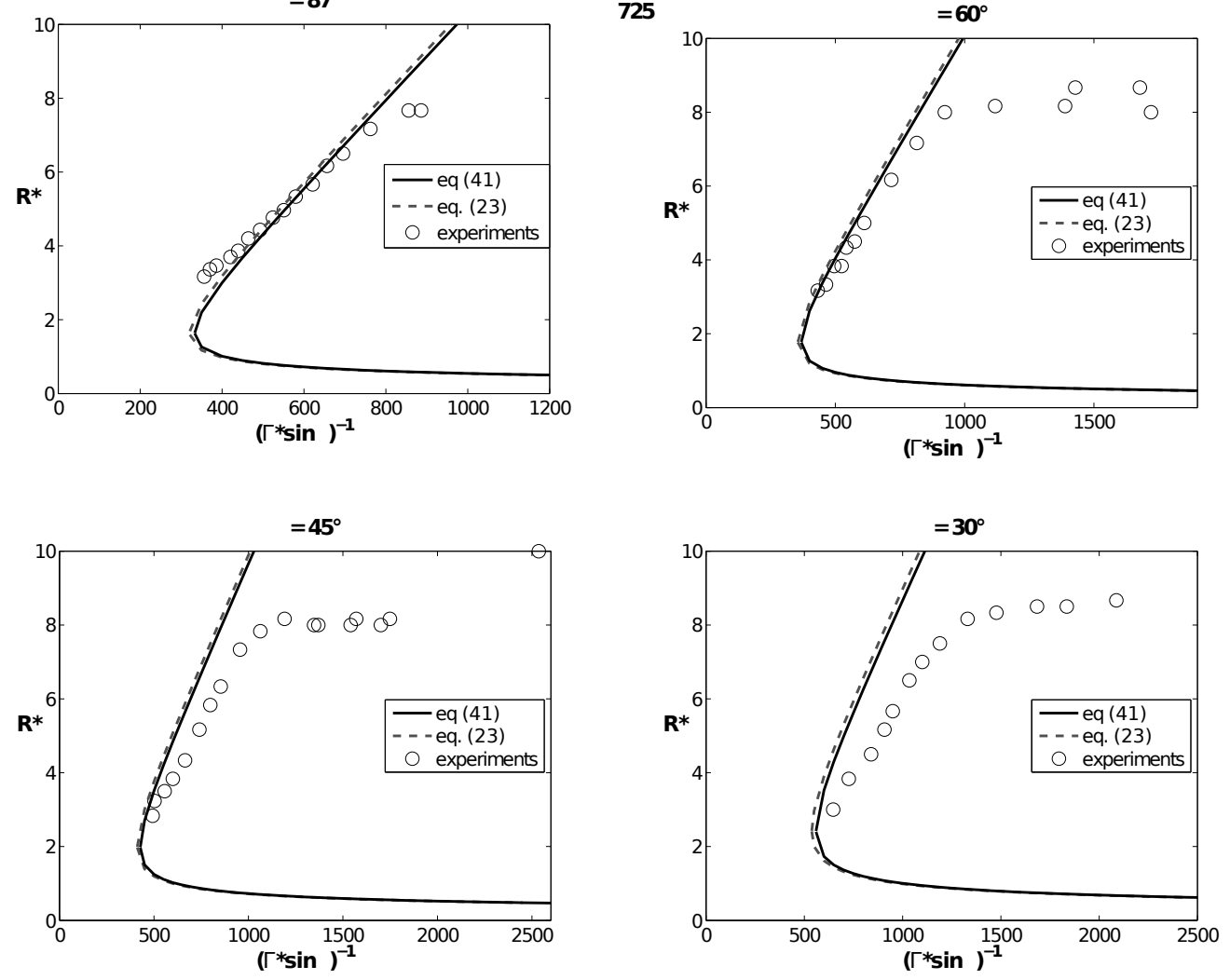

FIG. 19: Theory and experiments for the Rhodorsil 47V20 oil $\left(m=0.23\right.$ and $\left.\theta=52^{\circ}\right)$. As expected, the inertial effects do not play a significant role and both model 1 and model 2 give very similar predictions. These models provide a good agreement for high plate slope $\alpha>45^{\circ}$ and discrepancies for small plate slope.

experimental flow rate for both Huber and Rhodorsil 47V5 oils. Then, long waves should appear in the liquid film surrounding the dry patch and can introduce time modulation of the contact angle of the rim with a non zero mean value which will result in a change the evolution of the curvature radius at the apex with flow rate.

\section{Predicting rewetting}

As in the previous section, one can rewrite (41) with a dimensionless force $\tilde{F}_{i}$ that tends to open the dry patch if positive and consider its derivative with respect to the radius of 

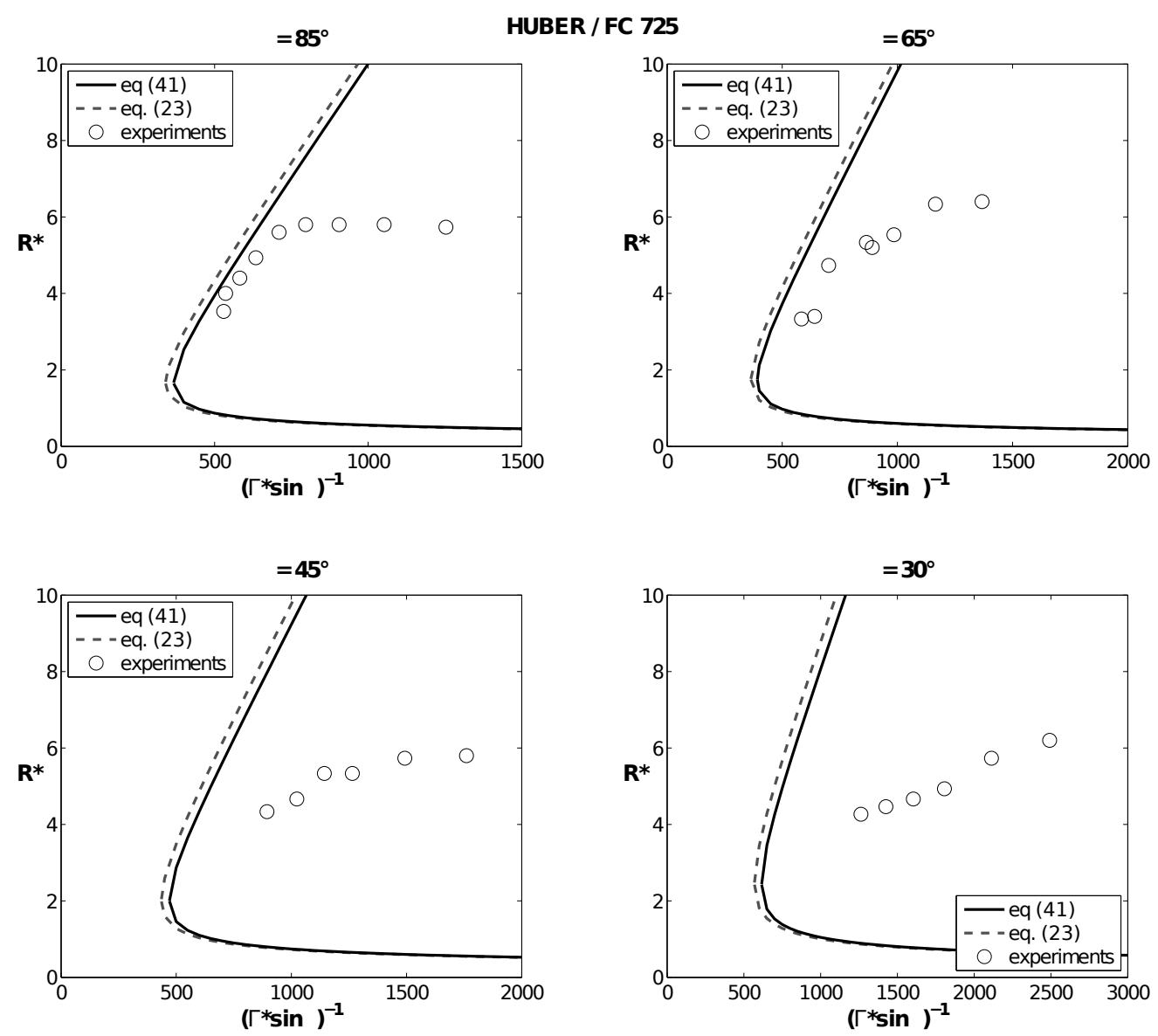

FIG. 20: Theory and experiments for Huber oil $\left(m=0.23\right.$ and $\left.\theta=52^{\circ}\right)$. For this oil the inertial effects change the prediction, which results in a model 2 slightly closer to experiments than model 1, but this effect seems negligible. The agreement is good for high plate slope $\left(\alpha>65^{\circ}\right)$ but becomes worse and worse when the plate slope decreases.

curvature at the apex $d \tilde{F}_{i} / d R *$. Then the limit of existence of the dry patch is given by :

$$
\begin{aligned}
\tilde{F}_{i} & =0 \\
\frac{d \tilde{F}_{i}}{d R^{*}} & =0
\end{aligned}
$$

The comparison between this prediction and our experimental results is given fig 23 where the previous prediction (28) is also recalled. As one can see, these effects of inertia on the flow inside the rim do not change significantly the prediction for the Rhodorsil 47V20 oil 

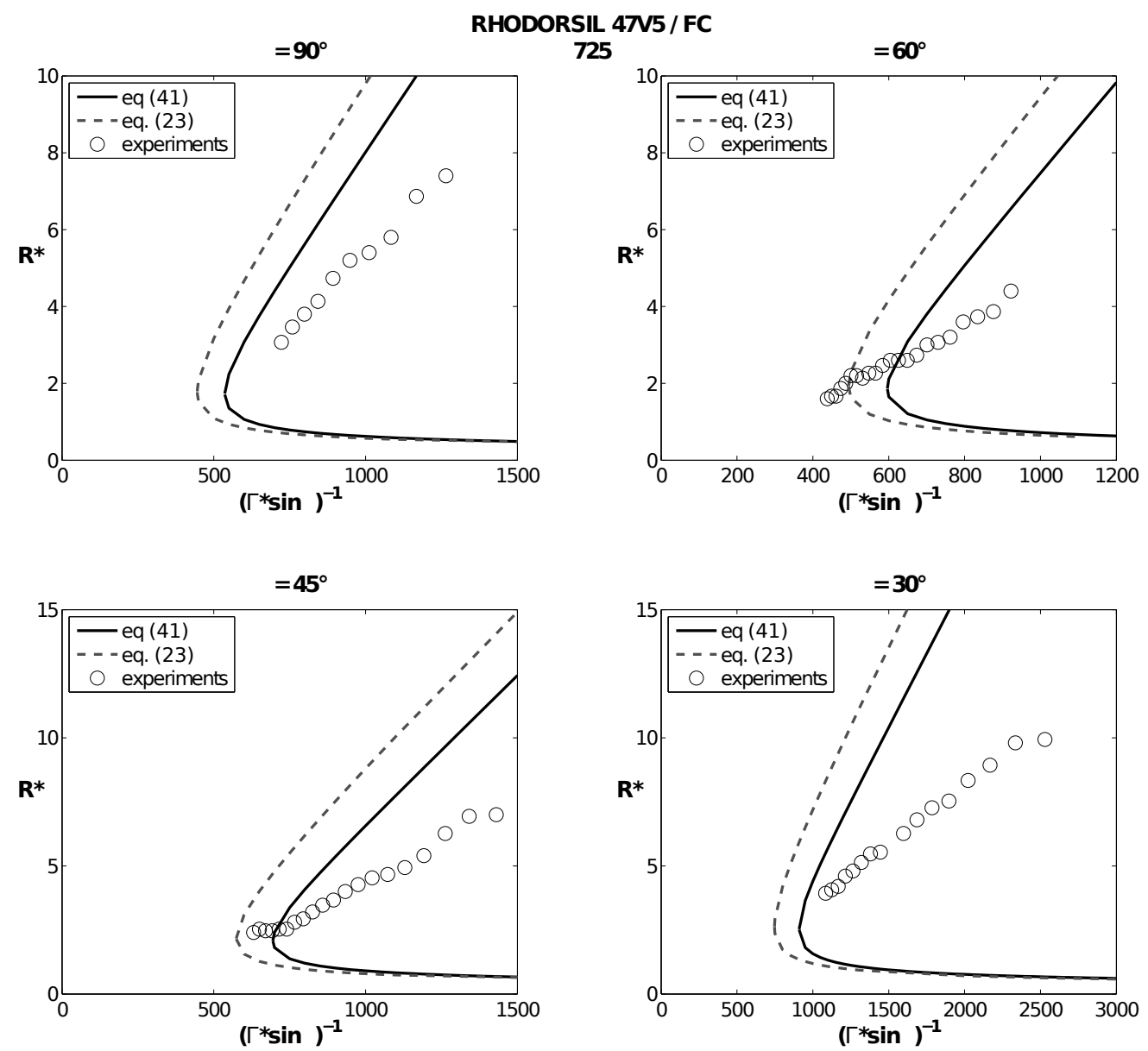

FIG. 21: Theory and experiments for the Rhodorsil 47V5 oil $\left(m=0.23\right.$ and $\left.\theta=52^{\circ}\right)$. In this case the inertial effects play a significant role and a large difference between model 1 and model 2 is observed. Model 2 clearly improves the prediction even if the agreement is good only for almost vertical plate.

where the agreement is good. For the Huber and Rhodorsil 47V5 oils, these inertial effects clearly greatly improve the prediction of the critical flow rate $\Gamma_{c}$, even if our prediction is still higher than the experimental value. 


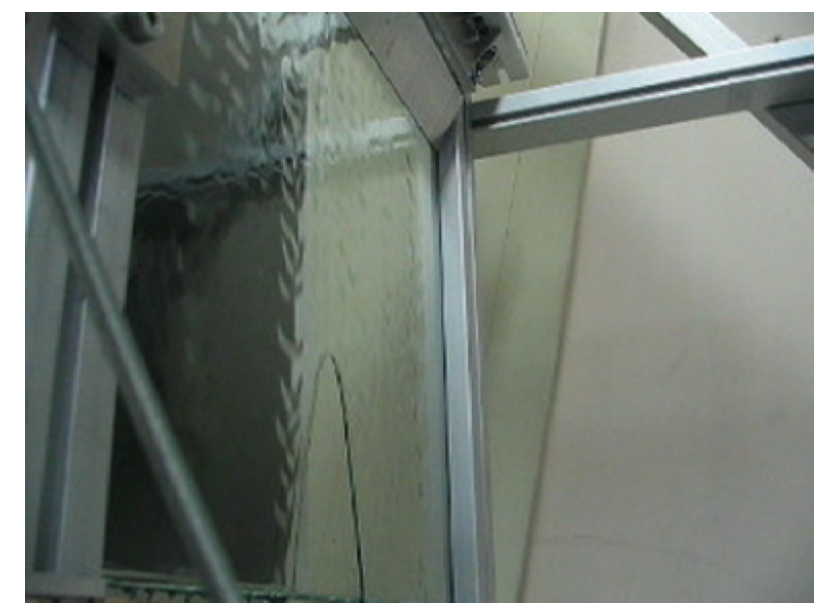

FIG. 22: Capillary waves in the liquid film around the dry patch (Rhodorsil 47V5 oil)
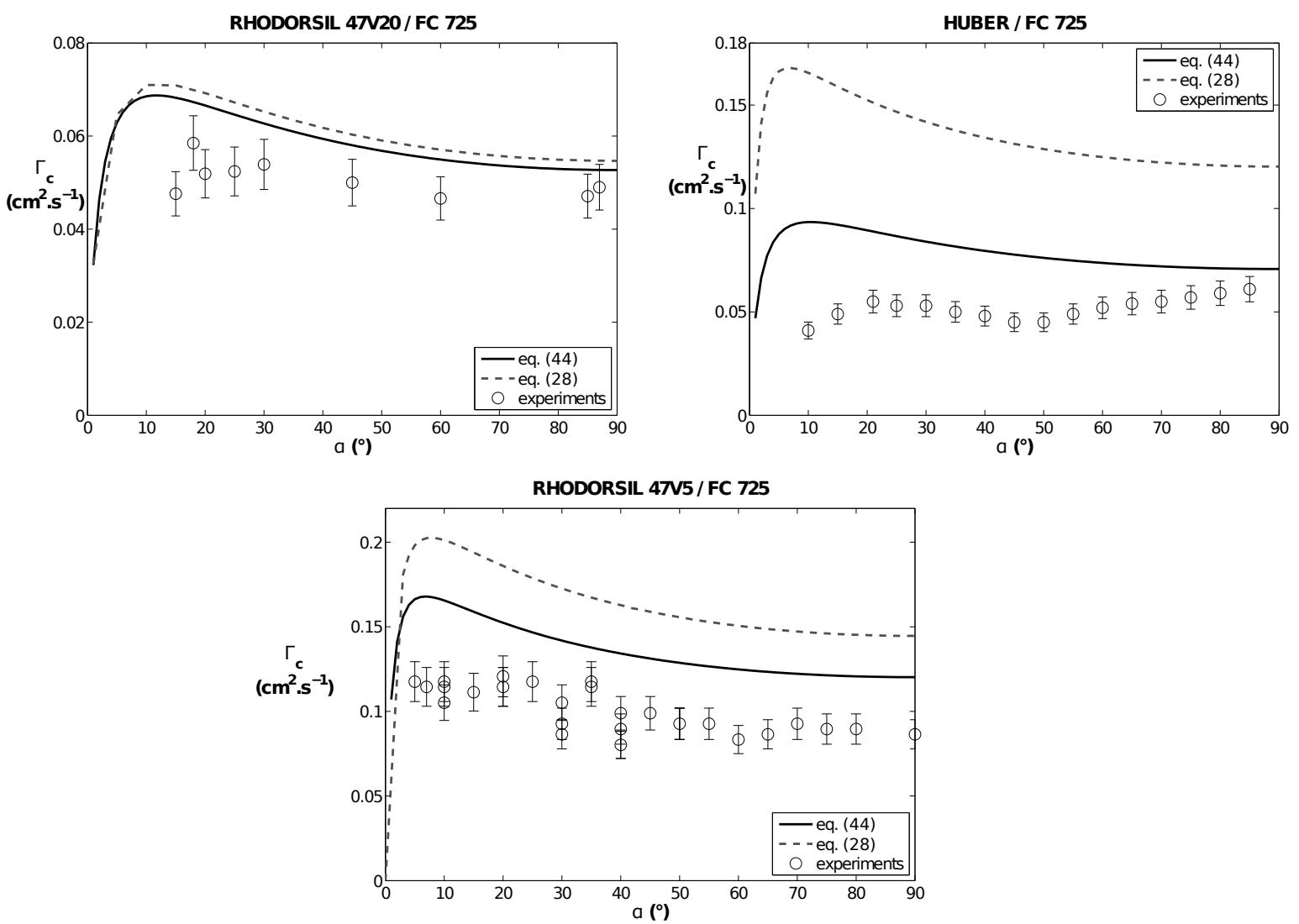

FIG. 23: Comparisons of the measured critical flow rate to the predictions of eqs. (28) and (44). 


\begin{tabular}{|l|l|c|c|c|c|c|}
\hline Oil & Wave apparition & $\alpha=15^{\circ}$ & $\alpha=30^{\circ}$ & $\alpha=45^{\circ}$ & $\alpha=60^{\circ}$ & $\alpha=75^{\circ}$ \\
\hline \hline Rhodorsil 47V20 & $\Gamma_{w}\left(\mathrm{~cm}^{2} . \mathrm{s}^{-1}\right)$ & 3.45 & 1.6 & 0.925 & 0.53 & 0.25 \\
\hline Rhodorsil 47V20 & $\left(\Gamma_{w}^{*} \sin \alpha\right)^{-1}$ & 117.02 & 130.5 & 159.9 & 226.1 & 436.7 \\
\hline \hline Huber & $\Gamma_{w}\left(\mathrm{~cm}^{2} . \mathrm{s}^{-1}\right)$ & 2.11 & 0.98 & 0.57 & 0.33 & 0.15 \\
\hline Huber & $\left(\Gamma_{w}^{*} \sin \alpha\right)^{-1}$ & 266.6 & 297.3 & 364.1 & 514.9 & 994.8 \\
\hline \hline Rhodorsil 47V5 & $\Gamma_{w}\left(\mathrm{~cm}^{2} . \mathrm{s}^{-1}\right)$ & 0.97 & 0.45 & 0.26 & 0.15 & 0.07 \\
\hline Rhodorsil 47V5 & $\left(\Gamma_{w}^{*} \sin \alpha\right)^{-1}$ & 1543.8 & 1721.9 & 2108.9 & 2982.5 & 5761.7 \\
\hline \hline
\end{tabular}

TABLE V: Values of the flow rate $\Gamma_{w}$ and of the non dimensional parameter $\left(\Gamma_{w}^{*} \sin \alpha\right)^{-1}$ above which long waves appear in the flow.

\section{CONCLUSION}

In summary, we have reconsidered the problem of dry patch shape and stability when the flow inertia becomes non negligible. Several key results have been obtained:

1. Though the prediction was established in a highly viscous limit, the shape of a dry patch is well described by the solution introduced by Podogorski et al. [13], even when the fluid viscosity is reduced to a few cP.

2. This result is issued from experiment, but has also been proved by taking into account explicitly the dry patch shape when inertia is taken into account in both the film and the rim (Section IV).

3. On the other hand, the radius of curvature at the apex of the dry patch, as well as the critical flow rate $\Gamma_{c}$ above which the dry patch disappears (rewetting transition), depend appreciably on inertia when the viscosity is reduced to a few $\mathrm{cP}$.

4. The prediction of these two quantities requires to take into account the curvature of the contact line at the apex $R^{-1}$, as first realized by S.D.R Wilson [20]. However, in contrast with this reference, the complete treatment leads to two branches of solution, one stable ( Podgorski branch), the other unstable, the critical flow rate being reached when these two branches are merging. 
5. We have proposed two different calculation of $\mathrm{R}(\Gamma, \alpha)$ and of $\Gamma_{c}$. The first one (Section III) takes into account only the inertia carried by the flowing film, while the second (Section IV) also treats the inertial effects in the rim. The first is sufficient in the 20 $\mathrm{cP}$ case investigated long ago by Podgorski but the second is necessary for the two other oils.

6. Despite these efforts, the prediction is not perfect, especially for the $\mathrm{R}(\Gamma, \alpha)$ behavior. However, for the largest viscosity, model 1 as well model 2 gives predictions for $\Gamma_{c}$ that are accurate at $10 \%$, while model 2 , in the other limit $(5 \mathrm{cP})$ gives prediction accurate at $20 \%$. Considering the complexity of this problem that mix several effects superimposed, and the absence of adjustable parameter in our modeling, we believe that the present contribution is an important step for the prediction of the rewetting threshold.

It would be now important to explore in more details the effects of the long waves observed on the film at low viscosity, that are presumably the origin of the remaining discrepancies. This effect could be explored by careful optical measurements of the correlation between the wave amplitude and the effective contact angle of the rim. Finally, it would be also interesting to explore the dependance of the dry patch on the wetting properties of the plate. Our modeling gives explicitly predictions for the dependance upon contact angle but our experiments were locked to a contact angle of order $45^{\circ}$. The limit of poor wettability $\left(\theta \simeq 90\right.$ to $\left.180^{\circ}\right)$ is especially interesting for many technical applications.

\section{Acknowledgments}

We acknowledge T. Podgorski for providing us unpublished measurements and for helpful suggestions.

[1] P.-G. de Gennes, "Wetting: statics and dynamics" Rev. Mod. Phys., 57, 827 (1985)

[2] L. Leger and J.-F. Joanny, "Liquid spreading" Rep. Progr. Phys., 55, 431 (1992)

[3] D. Bonn, J. Eggers, J. Indekeu, J. Meunier and E. Rolley, "Wetting and spreading" Rev. Mod. Phys., 81, 739 (2009) 
[4] P.G. de Gennes and F. Brochard-Wyart and D. Quéré," Capillarity and wetting phenomena. Drops, bubbles, pearls, waves", Edited by Springer, (2004)

[5] R.V. Craster and O.K. Matar, "Dynamics and stability of thin liquid films" Rev. Mod. Phys., 81, $1131(2009)$

[6] R. Blossey, " Thin Liquid Films ”, Edited by Springer, (2012)

[7] E. B. Dussan V., "On the spreading of liquids on solid surfaces: Static and dynamic contact lines," Ann. Rev. Fluid. Mech., 11, 371 (1979).

[8] F. Brochard-Wyart, J.M. Di Meglio and D. Quéré, "Démouillage" etude du retrait d'un film liquide non mouillant déposé sur un plaque ou une fibre" C.R. Acad. Sc. Paris, 304, 553 (1987)

[9] F. Brochard-Wyart, C. Redon and C. Sykes "Démouillage des films liquides ultraminces" C.R. Acad. Sc. Paris, 314, 14 (1992)

[10] C. Redon, F. Brochard-Wyart and F. Rondelez, "Dynamics of dewetting" Phys. Rev. Lett., 66, $715(1991)$

[11] G.I. Taylor and D.H. Michael ,"On making holes in a sheet fluid" J. Fluid Mech., 58, 625 (1973)

[12] D. E. Hartley and W. Murgatroyd, "Criteria for the breakup of thin liquid layers flowing isothermally over solid surfaces," Int. J. Heat Mass Transfer, 7, 1003 (1964).

[13] T. Podgorski and J.-M. Flesselles and L. Limat, "Dry arches within flowing films" Physics of Fluids, 11, 845 (1999)

[14] T. Podgorski, "Ruissellement en conditions de mouillage partiel" $\mathrm{PhD}$ Thesis University Paris $6,(2000)$

[15] T. Podgorski, J..M. Flesselles and L. Limat, "Courbure de la frontière d'une zone sèche dans un film en coulement" C.R. Acad. Sc. Paris, 9, 1361 (2001)

[16] T. Podgorski, J.M. Flesselles and L. Limat, "Corners, Cusps, and Pearls in Running Drops", Phys. Rev. Lett., 87, 36102 (2001)

[17] E. Rio, A. Daer and L. Limat, "Probing with a laser sheet the contact angle distribution along a contact line" J. Colloid Interface Sci, 269, 164 (2004)

[18] E. Rio and L. Limat, "Wetting hysteresis of dry patches left inside a flowing film" Physics of Fluid, 19, $032105.1(2006)$

[19] J. Sebilleau, L. Lebon and L. Limat "Stability of a dry patch in a viscous flow" Eur. Phys. J. Special Topics, 166, 139 (2009) 
[20] S. D. R. Wilson, "The stability of a dry patch on a wetted wall", Int. J. Heat Mass Transfer , 17, $1607(1974)$

[21] S. K. Wilson, B. R. Duffy and S. H. Davis , "On a slender dry patch in a liquid film draining under gravity down an inclined plane", Euro. Jnl of Applied Mathematics, 12, 233 (2001)

[22] Y.M. Yatim, B. R. Duffy and S. K. Wilson, "Travelling-wave similarity solutions for steadily translating slender dry patch in a thin liquid film", Physics of Fluids, 25, 062013 (2013)

[23] H.E. Huppert, "The propagation of two dimensional and axisymmetric viscous gravity currents over a rigid horizontal surface", J. Fluid. Mech., 121, 43 (1982)

[24] S. M. Troian, E. Herbolzheimer, S. A. Safran and J. F. Joanny, "Fingering Instabilities of Driven Spreading Films", Europhysics Letters, 10, 25 (1989)

[25] R. Goodwin and G.M. Homsy, "Viscous flow down a slope in the vicinity of a contact line", Physics of Fluids A, 3, 515 (1991)

[26] T.B. Benjamin, "Wave dormation in laminar flow down an inclined plane", J. Fluid Mech., 2, 554 (1958) 